\title{
Evidence for Intermediate Polars as the Origin of the Galactic Center Hard X-ray Emission
}

Hailey, Charles J.; Mori, Kaya; Perez, Kerstin; Canipe, Alicia M.; Hong, Jaesub; Tomsick, John A.; Boggs, Steven E.; Christensen, Finn Erland; Craig, William W.; Fornasini, Francesca Total number of authors: 17

Published in:

Astrophysical Journal

Link to article, DOI:

10.3847/0004-637X/826/2/160

Publication date:

2016

Document Version

Publisher's PDF, also known as Version of record

Link back to DTU Orbit

Citation $(A P A)$ :

Hailey, C. J., Mori, K., Perez, K., Canipe, A. M., Hong, J., Tomsick, J. A., Boggs, S. E., Christensen, F. E., Craig, W. W., Fornasini, F., Grindlay, J. E., Harrison, F. A., Nynka, M., Rahoui, F., Stern, D., Zhang, S., \& Zhang, W. W. (2016). Evidence for Intermediate Polars as the Origin of the Galactic Center Hard X-ray Emission. Astrophysical Journal, 826(2), [160]. https://doi.org/10.3847/0004-637X/826/2/160

\section{General rights}

Copyright and moral rights for the publications made accessible in the public portal are retained by the authors and/or other copyright owners and it is a condition of accessing publications that users recognise and abide by the legal requirements associated with these rights.

- Users may download and print one copy of any publication from the public portal for the purpose of private study or research.

- You may not further distribute the material or use it for any profit-making activity or commercial gain

- You may freely distribute the URL identifying the publication in the public portal 


\title{
EVIDENCE FOR INTERMEDIATE POLARS AS THE ORIGIN OF THE GALACTIC CENTER HARD X-RAY
} EMISSION

\author{
Charles J. Hailey ${ }^{1}$, Kaya Mori ${ }^{1}$, Kerstin Perez ${ }^{2}$, Alicia M. Canipe ${ }^{1}$, Jaesub Hong ${ }^{3}$, John A. Tomsick ${ }^{4}$, Steven E. Boggs ${ }^{4}$, \\ Finn E. Christensen ${ }^{5}$, William W. Craig ${ }^{4,6}$, Francesca Fornasini ${ }^{4}$, Jonathan E. Grindlay ${ }^{3}$, Fiona A. Harrison ${ }^{7}$, \\ Melania Nynka ${ }^{1,8}$, Farid Rahoui ${ }^{9}$, Daniel Stern $^{10}$, Shuo Zhang ${ }^{1}$, and William W. Zhang ${ }^{11}$ \\ ${ }^{1}$ Columbia Astrophysics Laboratory, Columbia University, New York, NY 10027, USA; chuckh@astro.columbia.edu \\ ${ }^{2}$ Haverford College, 370 Lancaster Avenue, KINSC L109, Haverford, PA 19041, USA \\ ${ }^{3}$ Harvard-Smithsonian Center for Astrophysics, Cambridge, MA 02138, USA \\ ${ }^{4}$ Space Sciences Laboratory, University of California, Berkeley, CA 94720, USA \\ ${ }^{5}$ DTU Space-National Space Institute, Technical University of Denmark, Elektrovej 327, DK-2800 Lyngby, Denmark \\ ${ }^{6}$ Lawrence Livermore National Laboratory, Livermore, CA 94550, USA \\ ${ }^{7}$ Cahill Center for Astronomy and Astrophysics, California Institute of Technology, Pasadena, CA 91125, USA \\ 8 Department of Physics and Astronomy, University of California, Irvine, 4129 Frederick Reines Hall, Irvine, CA 92697, USA \\ ${ }^{9}$ European Southern Observatory, K. Schwarzschild-Strasse 2, D-85748 Garching bei München, Germany \\ ${ }^{10}$ Jet Propulsion Laboratory, California Institute of Technology, Pasadena, CA 91109, USA \\ ${ }_{11}$ NASA Goddard Space Flight Center, Greenbelt, MD 20771, USA \\ Received 2015 December 4; revised 2016 May 16; accepted 2016 May 19; published 2016 July 28
}

\begin{abstract}
Recently, unresolved hard (20-40 keV) X-ray emission has been discovered within the central 10 pc of the Galaxy, possibly indicating a large population of intermediate polars (IPs). Chandra and XMM-Newton measurements in the surrounding $\sim 50 \mathrm{pc}$ imply a much lighter population of IPs with $\left\langle M_{\mathrm{WD}}\right\rangle \approx 0.5 M_{\odot}$. Here we use broadband NUSTAR observations of two IPs: TV Columbae, which has a fairly typical but widely varying reported mass of $M_{\mathrm{WD}} \approx 0.5-1.0 M_{\odot}$, and IGR J17303-0601, with a heavy reported mass of $M_{\mathrm{WD}} \approx 1.0-1.2 M_{\odot}$. We investigate how varying spectral models and observed energy ranges influences estimated white dwarf mass. Observations of the inner $10 \mathrm{pc}$ can be accounted for by IPs with $\left\langle M_{\mathrm{WD}}\right\rangle \approx 0.9 M_{\odot}$, consistent with that of the CV population in general and the X-ray observed field IPs in particular. The lower mass derived by Chandra and XMM-Newton appears to be an artifact of narrow energy-band fitting. To explain the (unresolved) central hard X-ray emission $(\mathrm{CHXE})$ by IPs requires an X-ray $(2-8 \mathrm{keV})$ luminosity function (XLF) extending down to at least $5 \times$ $10^{31} \mathrm{erg} \mathrm{s}^{-1}$. The CHXE XLF, if extended to the surrounding $\sim 50 \mathrm{pc}$ observed by Chandra and XMM-Newton, requires that at least $\sim 20 \%-40 \%$ of the $\sim 9000$ point sources are IPs. If the XLF extends just a factor of a few lower in luminosity, then the vast majority of these sources are IPs. This is in contrast to recent observations of the Galactic ridge, where the bulk of the $2-8 \mathrm{keV}$ emission is ascribed to non-magnetic CVs.
\end{abstract}

Key words: Galaxy: center - novae, cataclysmic variables - X-rays: diffuse background

\section{INTRODUCTION}

Recently, the Nuclear Spectroscopic Telescope Array $(\mathrm{NUSTAR})$ discovered an unresolved central hard X-ray emission (CHXE) in the inner $\sim 8 \mathrm{pc} \times \sim 4 \mathrm{pc}$ of the Galaxy (Perez et al. 2015; hereafter KP15). This emission has a 20-40 $\mathrm{keV}$ luminosity of $2 \times 10^{34} \mathrm{erg} \mathrm{s}^{-1}$ and can be described by either thermal bremsstrahlung with $k T>35 \mathrm{keV}$ or a power law with photon index $\Gamma \approx 1.2-1.9\left(N(E) \sim E^{-\Gamma}\right)$. KP15 propose that this emission is due to either stellar origins, such as large populations of intermediate polars (IPs), low-mass $\mathrm{X}$-ray binaries (LMXBs), or millisecond pulsars, or diffuse origins, such as cosmic ray outflows from the supermassive black hole Sagittarius A* However, these explanations, apart from the IP interpretation, encounter difficulties. A sufficient LMXB population must have a long mean time between outbursts or very faint outburst states to evade Galactic Center (GC) monitoring campaigns (Degenaar et al. 2012). The requisite population of several thousand millisecond pulsars would conflict with the dominant IP interpretation of the Chandra point source population in the same region (Muno et al. 2004a; Pretorius \& Mukai 2014). A cosmic ray origin has been discussed in the recent work of Dogiel et al. (2015), but requires fine tuning of the diffusion parameters to reproduce the observed spatial extent. Other possible diffuse sources such as synchrotron radiation from magnetic filaments or low-surface brightness pulsar wind nebulae are not supported by Chandra (Johnson et al. 2009) and NUSTAR observations (Mori et al. 2015) or supernova birth rates in this region, respectively.

A natural origin of the CHXE could be a population of cataclysmic variables (CVs), in particular IPs. CVs are accreting white dwarf (WD) binary systems with short orbital periods ( $\lesssim 1$ day) and $\mathrm{X}$-ray emission due to accretion via Roche-lobe overflow from a late-type main sequence companion. Magnetic CVs, which have WD magnetic fields strong enough to distort the inner accretion disk, are particularly copious emitters of hard $(>5 \mathrm{keV}) \mathrm{X}$-rays. IPs are a type of magnetic $\mathrm{CV}$ that, compared to polars, have longer orbital periods and non-synchronized orbits. In IPs, infalling material is funneled onto the WD poles along magnetic field lines and heated to temperatures that scale with the WD mass. A standoff shock, with height that adjusts itself to give infalling material time to cool to the photosphere temperature, is the location of the highest temperature material. Below the shock, there exists a column of cooling material, exhibiting a range of temperatures. Some of the emitted X-rays are viewed through the accretion curtain, requiring an additional absorption term with some partial-covering fraction depending on the orientation of the IP with respect to the observer. X-rays incident on the WD 
or pre-shock material may also cause Compton reflection and fluorescent neutral $\mathrm{Fe}$ line emission. Thus the temperatures and masses obtained for a particular analysis can vary depending on the particular components included in the model.

Chandra surveys of the central $2^{\circ} \times 0.8$ (Wang et al. 2002) and XMM-Newton surveys of the central $\sim 50$ pc (Heard \& Warwick 2013; hereafter HW13) of the Galaxy have both revealed emission consistent with a large population of magnetic CVs. Chandra has identified $\sim 9000$ point sources (Muno et al. 2009) in the GC, with the faint sources well described by a temperature of $k T \approx 8 \mathrm{keV}$ (Muno et al. 2004a). This is consistent with an IP origin and is the same temperature observed in Chandra studies of the diffuse emission of the central $\sim 20$ pc (Muno et al. 2004b). XMM-Newton also observed a diffuse X-ray spectrum described by $k T \approx 8 \mathrm{keV}$ thermal emission (HW13) and, using accretion shock theory (Suleimanov et al. 2005; Yuasa et al. 2012), inferred that the mass of the WDs is $\sim 0.5 M_{\odot}$. The ratio of $2-10 \mathrm{keV}$ luminosity to stellar mass, $L / M$, increases as it approaches a radius of $4 \mathrm{pc}$ and is on average $\sim 4$ times larger than that of the Galactic ridge, implying an IP density $\sim 10^{2}-10^{3}$ times higher than the IP density per integrated stellar mass observed in the solar neighborhood.

Observations of the Galactic ridge and bulge with $R X T E$ (Revnivtsev et al. 2009), Suzaku (Yuasa et al. 2012), and INTEGRAL (Krivonos et al. 2007) have also established a large population of IPs. The hard X-ray emission traces the infrared (IR) distribution associated with stellar populations and is consistent with IP spectra. The mean WD mass derived by these observations, also obtained using a model of the cooling post-shock region (Suleimanov et al. 2005; Yuasa et al. 2012), ranges from 0.5 to $0.66 M_{\odot}$, with an IP density more consistent with that found in the solar neighborhood.

IPs are thus a logical candidate for the CHXE, given their abundance in the solar neighborhood and GC, bulge, and ridge; however, a population of IPs that could account for the CHXE would have peculiar characteristics. The CHXE temperature is much higher than that observed by Chandra and XMM-Newton in nearby regions, and than that observed by Suzaku $(k T \sim 15$ $\mathrm{keV}$ ) in the bulge. The mean WD mass obtained by KP15 for the CHXE is $M_{\mathrm{WD}}>0.9 M_{\odot}$, consistent with the mean mass of field IPs measured by INTEGRAL and XMM-Newton, $0.86 \pm 0.07 M_{\odot} \quad$ (Bernardini et al. 2012). The heavy IPs detected in the INTEGRAL IBIS sample have a mean $k T \approx 20 \mathrm{keV}$ and $\sim 20 \%$ have $k T \approx 30-50 \mathrm{keV}$ (Landi et al. 2009). However, the CHXE fits to a photon index of $\Gamma=1.2-1.9$, whereas the IBIS sample has a mean photon index of $\Gamma \approx 2.7$ and the Galactic ridge and bulge emission fits to $\Gamma \approx 2.1$ (Revnivtsev et al. 2006; Yuasa et al. 2012). Additionally, the CHXE has a spatial distribution that falls much more steeply from the GC than the $2-10 \mathrm{keV}$ emission of Chandra and XMM-Newton, implying many more massive B-star progenitors in the inner parsecs or a higher efficiency for binary formation or accretion.

Thus the NUSTAR measurements of the CHXE and the Chandra and XMM-Newton measurements of the surrounding region imply that two distinct IP populations coexist in the GC: one population constrained to the inner parsecs resembling heavy field IPs and another population filling the surrounding tens of parsecs composed of much lighter IPs. At further distance from the GC, the IPs measured in the ridge by Suzaku, with their much lower $L / M$ and $k T \approx 15 \mathrm{keV}$, perhaps imply
Table 1

NUSTAR Observations of TV Col, J17303, and the GC

\begin{tabular}{lccc}
\hline \hline ObsID & $\begin{array}{c}\text { Start Date } \\
(\text { UTC })\end{array}$ & $\begin{array}{c}\text { Exposure } \\
(\mathrm{ks})\end{array}$ & Target \\
\hline 30001002001 & 2012 Jul 20 & 154 & Sgr A* \\
30001002003 & 2012 Aug 04 & 77 & Sgr A* \\
30001002004 & 2012 Oct 16 & 50 & Sgr A* \\
30001020002 & 2014 May 11 & 49 & TV Col \\
80002013012 & 2014 Jun 14 & 49 & J17303 \\
\hline
\end{tabular}

Note. The exposure times listed are corrected for good time intervals.

yet another evolutionary origin (e.g., massive B stars for the CHXE and later-type stars for the Galactic bulge). Alternatively, perhaps the CHXE represents the hot tail of an IP distribution that is detected by NuSTAR in the central parsecs, where a large enough density of these IPs exists to be detected above the background.

Further modeling of the CHXE as a heavy IP population, resembling the field IPs studied by INTEGRAL, is hampered by the limitations of previous individual IP analyses. First, the majority of heavy IP spectral measurements have poorly constrained temperatures/masses, due to either limited statistics or limited observed energy range. Second, a wide variety of IP spectral models have been applied, introducing systematics in the derived temperatures and masses.

To address these issues we selected two IPs for detailed study, the moderate-mass TV Columbae (hereafter TV Col) and the relatively heavy IGR J17303-0601 (hereafter J17303). Using the large effective area and energy range of $N U S T A R$, we are able to assess how different spectral models and energy bands cause systematic variations in the mass and temperatures derived.

Our analysis of TV Col and J17303, and its implications for the IP interpretation of the CHXE, proceeds as follows. In Section 2 we introduce these two objects and describe the observations used and data reduction applied. After reviewing various spectral models for the X-ray emission from IPs in Section 3, we present the results of spectral analysis of the two IPs in differing energy bands in Section 4 and address the systematics associated with the WD mass measurements in Section 5. We apply these same models to the CHXE spectrum, both using narrow-band $(2-10 \mathrm{keV})$ XMM-Newton data and broadband (2-40 keV) XMM-Newton and NuSTAR data, in Section 6. In Section 7 we use these results to demonstrate that a population of IPs with mean WD mass of $M_{\mathrm{WD}} \approx 0.87 M_{\odot}$ can consistently account for the CHXE. We will also discuss the implications for a IP population in the GC and the Galactic ridge X-ray emission. We summarize our results in Section 8.

\section{NUSTAR OBSERVATIONS AND DATA REDUCTION}

NUSTAR observed TV Col and J17303 in 2014 May and June for $\sim 50 \mathrm{ks}$ each, as listed in Table 1. NuSTAR is the first hard X-ray focusing telescope in orbit, operating from 3 to $79 \mathrm{keV}$. It contains two co-aligned optic and detector focal plane modules (FPMA and FPMB), with an angular resolution of $58^{\prime \prime}$ half power diameter (18" full-width at half-maximum, FWHM) and an energy resolution (FWHM) of $\sim 400 \mathrm{eV}$ below $\sim 50 \mathrm{keV}$ and $\sim 900 \mathrm{eV}$ at $68 \mathrm{keV}$ (Harrison et al. 2013). Each IP was fully contained on one of the four detector chips throughout the full observation, with the IP placed along the 
Table 2

Ten Spectral Models Used for Fitting NUSTAR Spectra of the Two IPs

\begin{tabular}{|c|c|c|}
\hline Model Name & Description & XSPEC Model ${ }^{\mathrm{a}}$ \\
\hline $1 \mathrm{~T}$ & Single temperature optically thin thermal & apec+Gauss \\
\hline IPM & IP mass model & ipm \\
\hline 1T-PC & Partially absorbed 1T optically thin thermal & pcfabs*apec+Gauss \\
\hline IPM-PC & Partially absorbed IP mass model & pcfabs*ipm \\
\hline 1T-RFL & $1 \mathrm{~T}$ optically thin thermal with reflection & reflect*apec+Gauss \\
\hline IPM-RFL & IP mass model with reflection & reflect*ipm \\
\hline IPM-PC-RFL & Partially absorbed IP mass model with reflection & pcfabs*reflect*ipm \\
\hline 1T-IPM & Single temperature optically thin thermal plus IP mass model & apec+ipm \\
\hline 2T-PC & Partially absorbed 2T optically thin thermal & pcfabs* (apec+apec+Gauss) or apec+pcfabs*apec+Gauss \\
\hline 3T-PC & Partially absorbed 3T optically thin thermal & apec+pcfabs* (apec+apec+Gauss) \\
\hline
\end{tabular}

Note. $2 \mathrm{~T}$ models are fit with partial absorption of both APECs and of only one APEC. Thermal models are fit with and without iron lines.

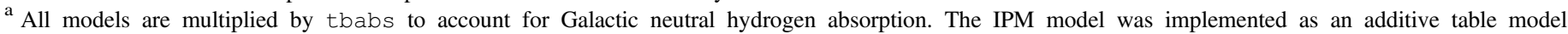
polarmodel.fits.

optical axis. For the CHXE analysis, we use observations of the Sagittarius $A^{*}$ region in 2012 , the three $N u S T A R$ observations listed in Table 1, and two archived XMM-Newton observations, 0694640301 and 0694641101, reprocessed with SAS v12.0.1. These are the same observations used in KP15. All NuSTAR data were processed using the NuSTAR Data Analysis Software (NuSTARDAS) v1.3.1.

After filtering for periods of high background during passage through the South Atlantic anomaly, we extracted source spectra from a $r=70^{\prime \prime}$ circular region centered at each source and generated $N u S T A R$ response matrix (rmf) and ancillary response (arf) files using nuproducts. Background spectra were extracted from an L-shaped region surrounding, and on the same detector chip as, the source. We grouped NuSTAR FPMA and FPMB spectra so that each bin had a source significance of $4 \sigma$ ( $3 \sigma$ for the last bin) above background. In joint FPMA and FPMB fits, we included a normalization factor in our fit models to account for the small cross-calibration difference, which is in all cases less than $2.6 \%$. All spectral fitting and flux derivations were performed in XSPEC (Arnaud 1996), with photoionization cross sections set to those from Verner et al. (1996) and the abundances for the interstellar absorption set to those from Wilms et al. (2000). Chi-squared statistics were used to assess spectral fitting and all quoted errors are at $90 \%$ confidence level. For both TV Col and J17303, we use energies $3-50 \mathrm{keV}$, above which the detector background is significant.

\section{OVERVIEW OF IP SPECTRAL MODELS}

Several spectral models were applied in order to assess the systematics introduced on the derivations of temperature and mass. These models include primary X-ray emission from the shocked accretion column (Section 3.1) and photon reprocessing via partial absorption by the accretion curtain and/or reflection from the WD surface (Section 3.2). Table 2 shows a summary of the spectral models, and Figure 1 illustrates the regions contributing to each emission mechanism.

\subsection{Primary X-Ray Emission from the Shocked Accretion Column}

The simplest IP spectral model describes the emission from the accretion column as an optically thin thermal plasma in collisional ionization equilibrium. For this, we use the APEC model (Smith et al. 2001), hereafter referred to as the onetemperature or 1T model. This is clearly an approximation, since the accretion column in the post-shock region is continuously cooling. The derived average, or color, temperature is thus a lower limit to the shock temperature.

The most physical IP models utilize one-dimensional accretion flow models that follow material through the transition shock and down to the WD surface. They use either simple emissivity profiles ignoring the soft X-ray line region (Suleimanov et al. 2005) or a more detailed soft X-ray emissivity profile (Yuasa et al. 2010). We will use the IP mass (IPM) model (Suleimanov et al. 2005), which derives a density and temperature profile of the post-shock accretion column and integrates the resultant bremsstrahlung emission as a function of distance from the shock. The key parameters of this model are the shock temperature and the WD mass, since the emission depends on the depth of the gravitational potential well, assuming the relation between WD mass and radius of Nauenberg (1972).

The disadvantage of the IPM model is that it accounts only for the continuum bremsstrahlung emission (although it does include line cooling) and neglects the ionized line emission, including the prominent Fe lines. Acceptable fits to the IPM model can be obtained by ignoring the $5.5-7.5 \mathrm{keV}$ energy bins where $\mathrm{H}$-like and He-like $\mathrm{Fe}$ lines are found (Ezuka \& Ishida 1999). These fits improve with increasing temperature, since the relevant lines become near or fully ionized and thus very weak. For instruments with a broadband energy response, such as NUSTAR, this is sufficient to characterize the WD mass, as shown below. This is equally true for the $1 \mathrm{~T}$ model, where ignoring the line-producing region is equivalent to fitting a color temperature to the broadband bremsstrahlung.

We also consider a two-temperature (or 2T) model, described by two APEC components. This will allow comparison with previous analyses of both individual IPs and the GC populations. Although not made clear in the literature, this $2 \mathrm{~T}$ model is strongly motivated as an approximation to the one-dimensional accretion flow models and it is supported by our simulation where we used the NUSTAR responses for consistency with the subsequent spectral analysis. We simulated a 3-50 keV NuSTAR spectrum using the IPM model with $M_{\mathrm{WD}}=0.8 M_{\odot}$ and partial-covering absorption (described in Section 3.2) with $N_{\mathrm{H}}=5.0 \times 10^{23} \mathrm{~cm}^{-2}$, corresponding to previous measurements of TV Col. When fit with a 2T model, a low temperature of $k T_{\text {low }} \approx 5 \mathrm{keV}$ and a high temperature of $k T_{\text {high }} \approx 22 \mathrm{keV}$ are obtained, along with the correct column density. Similarly, a simulated spectrum using the IPM model 


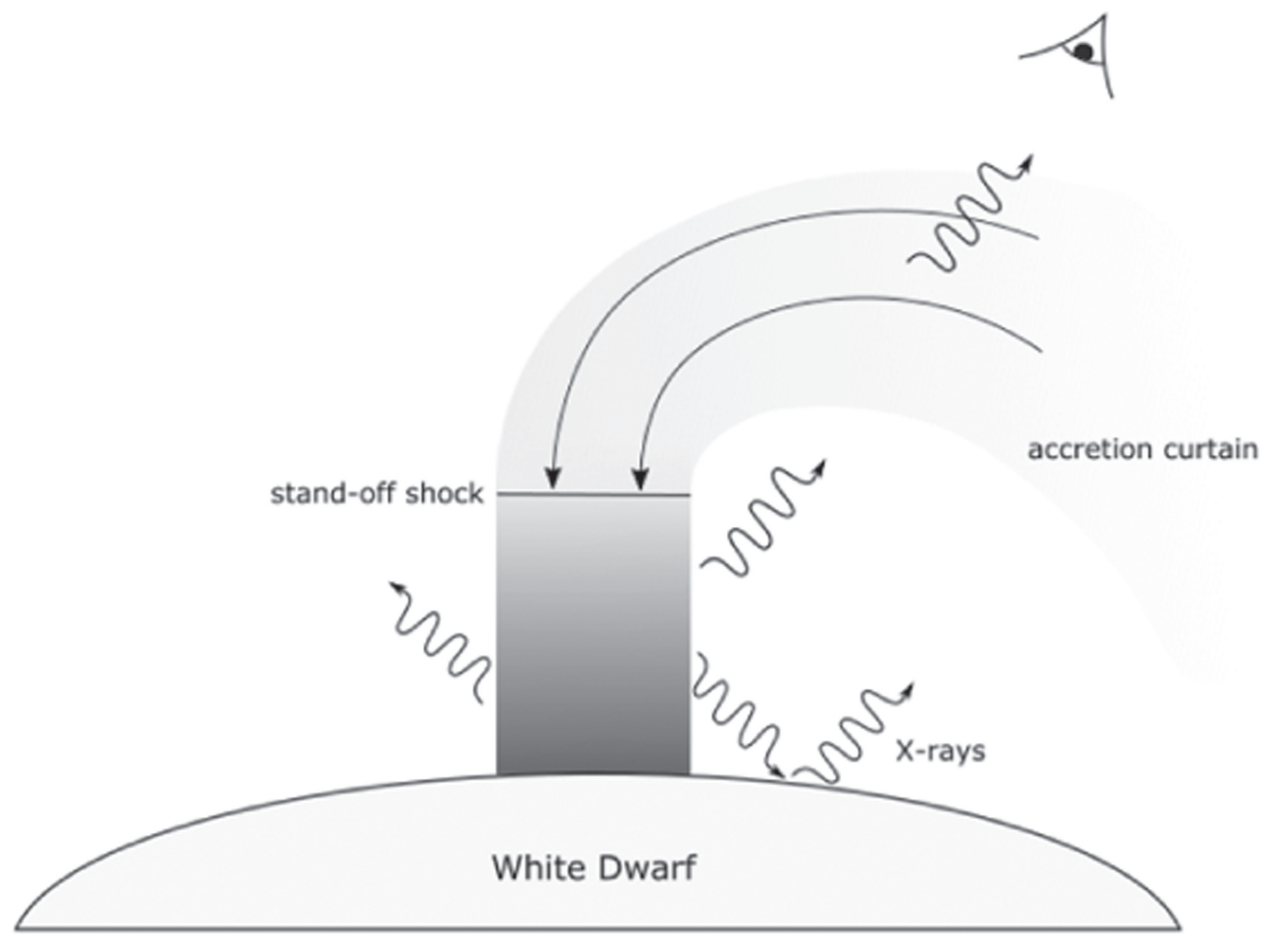

Figure 1. A schematic view of IP geometry and X-ray emission regions. Primary thermal X-ray photons emitted from the shocked accretion column can be absorbed by the accretion curtain or reflected from the WD surface. Along the post-shock accretion column, plasma density increases toward the WD surface as indicated by the color gradient in the figure.

with $M_{\mathrm{WD}}=1.0 M_{\odot}$ and $N_{\mathrm{H}}=2.2 \times 10^{24} \mathrm{~cm}^{-2}$, corresponding to previous measurements of $\mathrm{J} 17303$, is well fit by a $2 \mathrm{~T}$ model with $k T_{\text {low }} \approx 5 \mathrm{keV}$ and a slightly hotter high temperature of $k T_{\text {high }} \approx 30 \mathrm{keV}$. In both cases, deviations between the simulated IPM spectra and the best-fit 2T models are mostly within $5 \%$ while they increase up to $\sim 10 \%$ only at $E \sim 50 \mathrm{keV}$. Depending on the details of instrument response and whether Fe lines are included in the spectra, the lowtemperature component varies from $k T_{\text {low }} \approx 3 \mathrm{keV}$ to $8 \mathrm{keV}$ for $M_{\mathrm{WD}} \sim 0.5-1.0 M_{\odot}$ IPs. This $2 \mathrm{~T}$ phenomenological model is further supported on theoretical grounds. The high-temperature component from the immediate vicinity of the transition shock dominates hard X-ray emission thus it closely tracks the shock temperature which is proportional to the WD mass, while the low-temperature component originates from near the bottom of the accretion column. A plot of the thermal bremsstrahlung emissivity $\sim \rho^{2} T^{1 / 2}$ (where $\rho$ and $T$ are density and temperature, respectively) from Yuasa et al. (2010) shows a peak in emissivity at $k T \lesssim 10 \mathrm{keV}$ for massive IPs, which can account for the low-temperature component.

Another primary X-ray emission model used by previous studies associates the low-temperature component of the $2 \mathrm{~T}$ model with an ionization temperature through emission line ratio fitting. For most IPs, a ratio of $\mathrm{He}$-like and $\mathrm{H}$-like $\mathrm{Fe}$ emission lines at 6.7 and $6.9 \mathrm{keV}$ can be used as a temperature diagnostics for thermal plasma in ionization equilibrium (Mewe et al. 1985). Utilizing a large sample of IPs, ASCA obtained ionization temperatures of $\sim 7-13 \mathrm{keV}$, with a mean of $k T_{\text {ion }}=8.1 \mathrm{keV}$ (Ezuka \& Ishida 1999). This temperature correlates well with the lower of the temperatures in the twotemperature fits, and corresponds to the region of peak line cooling. The ASCA high temperatures were crudely estimated to be $\sim 15-30 \mathrm{keV}$, far above the ASCA energy band, but consistent with the continuum temperatures observed in joint XMM-Newton and INTEGRAL fits to an ensemble of IPs, $\sim 15-40 \mathrm{keV}$ (Landi et al. 2009). In NuSTAR broadband data, however, we will see that small changes to the hightemperature component can cause large changes to the parameters obtained for the low-temperature component.

If we fit the NuSTAR data in the narrow energy band of 3-10 $\mathrm{keV}$ to a single effective ionization temperature, this largely decouples the spectral fitting in the soft X-ray band from large variations introduced by an additional temperature associated with the hard X-ray continuum. Moreover, exploiting this approach will afford some insight into previous interpretation of ASCA IP spectral fitting and to the XMM-Newton fits to the CHXE, discussed in Section 6. We call this an effective ionization temperature because it is a temperature determined largely from line ratios, but since the accretion column is continually cooling toward the surface, the temperature is only defined in a mean sense. However as discussed above, the emissivity will have a maximum at $k T \lesssim 10 \mathrm{keV}$, so that we expect a single temperature fit should adequately represent the line structure. Then using a model relating effective ionization temperature and WD mass (Aizu 1973), we should recover the WD mass obtained from our broadband energy fits.

\subsection{X-Ray Reprocessing by the Accretion Curtain and WD Surface}

As mentioned in Section 1, phase-resolved spectroscopy provides evidence for an accretion curtain. In phase-averaged observations, this manifests itself as an absorption component that is local to the WD and covers part of the emitting region, and is in addition to the usual interstellar absorption. The exact location of the curtain is unclear, but given that it is neutral or warm-ionized, a likely location is above the transition shock. 
Most of the models we present below include a partial-covering absorption term, using the pcfabs model in XSPEC with neutral hydrogen column density $N_{\mathrm{H}}^{\mathrm{pc}}$ and covering fraction $f^{\mathrm{pc}}$, as a necessary component to obtain good fits.

The most physically plausible model should also account for $\mathrm{X}$-ray reflection off the WD surface. X-rays from the cooling accretion column will reflect from this surface, leading to both Compton reflection of the hard X-rays and neutral or nearly neutral $\mathrm{Fe}-\mathrm{K}$ fluorescence and absorption features. While reflection-like features will be produced by cold or warm absorbing material in the pre-shock region, such material may or may not be present; but reflection from the surface should always be present, since the transition shock is located close enough to the surface that the solid angle for interception of downward radiation is approximately unity. Neutral Fe features with typical equivalent widths of $\approx 100-200 \mathrm{eV}$ are prominent in many IPs (Ezuka \& Ishida 1999). Evidence of reflection from WD surfaces has been tentatively claimed using $A S C A$ data (Ezuka \& Ishida 1999) and definitively seen using NuSTAR data (Mukai et al. 2015).

To model X-ray reflection from the WD surface, we use the convolution model reflect (Magdziarz \& Zdziarski 1995) for reprocessing the primary X-ray continuum and a Gaussian line at $6.4 \mathrm{keV}$ for the neutral $\mathrm{Fe}$ line. TV $\mathrm{Col}$ is a particularly attractive candidate for reflection modeling because the observation angle between its rotation axis and the Earth has been well constrained by previous observations. Unfortunately, the partial-covering model (PC model) we apply can also account for some of the X-ray reflection effects, as pointed out by Yuasa et al. (2010). This will lead to considerable parameter degeneracy between the two models. We note that Cropper et al. (1998) found that the inclusion of a reflection component along with the PC model did not significantly change the derived WD mass. However, given the combination of good statistics and broadband coverage of NuSTAR, additional investigation seems warranted.

\section{SPECTRAL ANALYSIS OF TV COL AND J17303}

Previous studies of field IPs have often exhibited large discrepancies in the continuum temperatures and WD masses due to using different energy bands and spectral models. In order to compare our results directly with the previous temperature/mass measurements and assess their systematic errors (Section 5), we fit a variety of spectral models shown in Table 2, including the simplest 1T model, to different combinations of the primary emission (1T, 2T, and IPM) and reprocessing models (PC and RFL), to NuSTAR data of TV Col and $\mathrm{J} 17303$. We also determine the ionization temperature by fitting a $1 \mathrm{~T}$ model with partial covering in the $3-10 \mathrm{keV}$ band and our results are discussed in Section 5.1 in comparison with the ASCA results by Ezuka \& Ishida (1999). We apply two different approaches to fit the IPM model to measure the WD mass. First, we fit the full 3-50 keV energy band, excluding Fe lines at 5.5-7.5 keV, with an IPM model with partial-covering (PC model) and/or X-ray reflection from the WD surface (RFL model). Second, we fit the NuSTAR data above $E \sim 15 \mathrm{keV}$ with an uncovered IPM model. Further discussions on the WD mass measurements can be found in Section 5.2. We present figures and tables associated with our fitting results in the Appendix.

\subsection{TV Col}

TV Col was first discovered in X-rays by Ariel 5 (Cooke et al. 1978), and subsequently observed by ROSAT (Vrtilek et al. 1996), RXTE (Rana et al. 2004), Swift (Brunschweiger et al. 2009), and Suzaku (Yuasa et al. 2010). It is located nearby $(d=368 \mathrm{pc})$, with an associated $V \sim 13$ late-type dwarf companion (McArthur et al. 2001) and fairly typical spin and orbital periods (spin period $P_{\text {spin }} \approx 1.9 \mathrm{ks}$, orbital period $P_{\text {orb }} \approx 5.5 \mathrm{hr}$, and $\left.P_{\text {spin }} / P_{\text {orbit }} \approx 0.1\right)$. It exhibits both positive and negative superhumps (Retter et al. 2003). It has shown optical outbursts of approximately two orders of magnitude, which have been associated with increased mass transfer from the companion (Hellier \& Buckley 1993). Analysis of line emission during the outbursts suggests it comes from the impact of the accretion stream with the outer parts of a precessing accretion disk. The observed orbit phase-resolved spectroscopy and energy-dependent power spectral analysis have been interpreted to suggest primarily disk-fed accretion or accretion from both the disk and stream (Rana et al. 2004), which is well described by the accretion curtain model with the observer at a modest inclination angle (Rosen et al. 1988). A range of temperatures, $k T \approx 20-40 \mathrm{keV}$, have been deduced for TV Col, depending on the particular form of the spectral model applied. While broad, this temperature range is consistent with the majority of IPs observed by INTEGRAL surveys (Landi et al. 2009).

\subsubsection{Thermal Plasma Model Fitting}

The 1T models without partial covering give a reduced $\chi^{2}=1.33$ with $k T=20.7 \pm 0.4 \mathrm{keV}$ (top left panel of Figure 3). However, phase-resolved studies suggest that an accretion curtain is present and thus a PC model should be used (Rana et al. 2004). The 1T-PC gives a lower temperature of $k T=15.6 \pm 0.4 \mathrm{keV}$ and $\chi_{\nu}^{2} \approx 1.2$ (top right panel of Figure 3 ). The 1T-RFL model gives a similar temperature of $k T=16.7_{-1.9}^{+0.7} \mathrm{keV}$ and $\chi_{\nu}^{2} \approx 1.2 .^{12}$ We additionally fit the narrow-band NUSTAR 3-10 keV data with a $1 \mathrm{~T}$ model with partial covering, to obtain an effective ionization temperature of $k T_{\text {ion }}=9.8 \pm 0.9 \mathrm{keV}$.

The temperature derived by Swift/BAT Brunschweiger et al. (2009), using a 1T bremsstrahlung model without partial covering, agrees with the temperature we derive using the $1 \mathrm{~T}$ model ignoring the line emission region (in which case the APEC closely approximates a bremsstrahlung). Excluding the 5.5-7.5 keV line emission region, as was done by Ezuka \& Ishida (1999), leads to systematically higher temperatures and better fits for 1T models, regardless of whether the model has a partial covering or reflection. This is readily understandable, since a high temperature will fit the hard X-ray emission from near the transition shock, but will over-ionize the line emission region, which requires a lower temperature in the cooling accretion column.

Fitting an uncovered 2T model and a 2T-PC model over both temperature components yields $\chi_{\nu}^{2}=1.2$ and 1.1 , respectively (two middle panels in Figure 3). A partial covering over both temperature components is a physically plausible representation of an accretion curtain where the neutral material may be above the transition shock. The best-fit value of $k T_{\text {low }} \approx 2-3$

\footnotetext{
12 Note that the observation angle for the RFL model is frozen to the value $\cos (\theta)=0.3$, corresponding to an $\sim 70^{\circ}$ inclination angle, measured previously using phase-resolved data (Rana et al. 2004).
} 
$\mathrm{keV}$ corresponds closely to the temperature at the base of the accretion column for IPs simulated with $M_{\mathrm{WD}}=0.8 M_{\odot}$ and it is consistent with theoretical expectations discussed in Section 3.1. The PC model gives hard X-ray temperatures of $k T_{\text {high }} \approx 20 \mathrm{keV}$. The best-fit $k T_{\text {high }}$ and $k T_{\text {low }}$ remain consistent whether only one or both temperature components are partially covered.

The best-fit interstellar absorption column densities are higher than the value obtained by Rana et al. (2004): $N_{\mathrm{H}}^{f}=2.1_{-0.1}^{+0.2} \times 10^{20} \mathrm{~cm}^{-2}$. The low column density of Rana et al. (2004) is based on jointly fitting ROSAT and RXTE spectra of TV Col from $0.1 \mathrm{keV}$ to $20 \mathrm{keV}$ and it is consistent with the source distance of $368_{-15}^{+17}$ pc (McArthur et al. 2001). The discrepancy is due to the lack of low-energy data below 3 $\mathrm{keV}$ for our NuSTAR analysis as Yuasa et al. (2010) also measured a similarly high column density $\left(N_{\mathrm{H}}^{f}=3.4_{-2.6}^{+1.3} \times 10^{22} \mathrm{~cm}^{-2}\right)$ by fitting the $3-50 \mathrm{keV}$ Suzaku spectra of TV Col. In addition, $N_{\mathrm{H}}^{f}$ is poorly constrained for some models with partial covering largely due to the degeneracy between $N_{\mathrm{H}}^{f}$ and $N_{\mathrm{H}}^{p c}$. To investigate the parameter sensitivity to $N_{\mathrm{H}}^{f}$, we refitted the NUSTAR spectra of TV Col by fixing $N_{\mathrm{H}}^{f}$ to the value of Rana et al. (2004) for all the models presented in Table 7. Both the temperature and the WD mass of TV Col remained unchanged within the errors. Therefore, we conclude that our main results and interpretations based on the temperatures and WD masses are not affected by the systematic errors associated with the interstellar absorption.

Sub-solar abundances and equivalent widths in the $\sim 100-200 \mathrm{eV}$ range, consistent with all the models shown, are observed for most field IPs. The $r e l_{\text {refl }}$ parameter measures the angle-averaged fraction of downward radiation intercepted by the WD surface, and the high values seen here are consistent with the theoretical expectation that the transition shock is extremely close to the surface (Yuasa et al. 2010). The statistical uncertainties in the derived temperatures and WD masses are small. The systematic error, due to different applied models, has not been fully considered by previous observers. Averaging the models here yields a mean temperature of $k T=21 \mathrm{keV}$, with a systematic uncertainty of $\sim 3.5 \mathrm{keV}$ estimated as the spread in $k T$ obtained from different $1 \mathrm{~T}$ models. The $2 \mathrm{~T}$ model produces substantially lower values of partial covering column densities compared to the $1 \mathrm{~T}$ model, and the RFL model does not require any partial covering component to produce acceptable fits.

\subsubsection{IPM Model Fitting}

Using the full 3-50 keV band, the IPM-PC and the IPM-RFL model yield consistent WD masses of $M_{\mathrm{WD}}=0.80_{-0.02}^{+0.01} M_{\odot}$ and $M_{\mathrm{WD}}=0.78 \pm 0.03 M_{\odot}$, respectively. Even with the much higher sensitivity and counting statistics of the NuSTAR observations, there is no definitive evidence that a RFL model is preferred. To the contrary, the PC model seems almost completely degenerate with the RFL model, producing comparable fit quality, abundances, temperatures, and masses. Thus we do not report results for a model with both reflection and partial covering. The masses in our IPM-PC model are consistent with the Suzaku measurements $\left(M_{\mathrm{WD}}=0.91_{-0.10}^{+0.14} M_{\odot}\right)$ of Yuasa et al. (2010) using a one-dimensional accretion flow model (called IPPSR) including both the continuum and emission lines. Thus $N U S T A R$ broadband energy measurement in which line emission is ignored produce WD masses that are consistent with those obtained by various observatories.

We also fit the $15-50 \mathrm{keV} \mathrm{NuSTAR}$ data with an uncovered IPM model and it yields a good $\chi_{\nu}^{2}=0.96$ since the effect of partial covering is negligible at such high energies. The best-fit WD mass of $M_{\mathrm{WD}}=0.77 \pm 0.03 M_{\odot}$ from the $E>15 \mathrm{keV}$ fit is in good agreement with the $3-50 \mathrm{keV}$ band IPM-PC fit results $\left(M_{\mathrm{WD}}=0.80_{-0.02}^{+0.01} M_{\odot}\right.$ and $\left.\chi_{\nu}^{2}=1.14\right)$ as well as the masses derived by Swift/BAT $\left(M_{\mathrm{WD}}=0.78 \pm 0.06 M_{\odot}\right.$, 14-195 keV; Brunschweiger et al. 2009) and by Suzaku $\left(M_{\mathrm{WD}}=0.87_{-0.18}^{+0.53} M_{\odot}, 15-40 \mathrm{keV}\right.$; Yuasa 2013) using an IPM model without partial covering.

\section{2. $J 17303$}

Unlike TV Col, J17303 has extreme properties for an IP with a spin period $P_{\text {spin }}=128 \mathrm{~s}$, orbital period $P_{\text {orb }} \approx 924$ minutes, and $P_{\text {spin }} / P_{\text {orbit }} \approx 2 \times 10^{-3}$. It has the second shortest spin to orbital period ratio of any known IP (Scaringi et al. 2010). The multiple emission components can be modeled by several optically thin thermal plasmas, with evidence for several absorbing components, including a warm (O VII) absorber that suggests possible photoionization of pre-shock material. The NuSTAR hard X-ray spectrum, discussed below, is also peculiar, showing evidence of reprocessing from one or more sites around the WD. Observations have consistently yielded a temperature of $k T \approx 60 \mathrm{keV}$, placing J17303 among the hottest in the Suzaku survey. Due to its brightness and extremely hard X-ray spectrum, J17303 is the optimal IP to compare the NuSTAR measurement of temperature and WD mass with the Swift (Brunschweiger et al. 2009) and INTEGRAL (Landi et al. 2009) results that extends to higher energy than NUSTAR.

\subsubsection{Thermal Plasma Model Fitting}

Unlike in the case of TV Col, a 1T model without partial covering or reflection shows large residuals in the $10-40 \mathrm{keV}$ energy band and a high $\chi_{\nu}^{2}=1.90$ (upper left panel of Figure 4 in the Appendix). Such a high-energy "hump" can result from strong reflection from the WD surface, but could also be produced by strong absorption in an accretion curtain. A 1T-PC model gives an excellent $\chi_{\nu}^{2}=1.05$, even including the Fe line-emitting 5.5-7.5 $\mathrm{keV}$ region (upper right panel in Figure 4), and yields $k T=26 \pm 1 \mathrm{keV}$, which is typical of many individual IPs even though $\mathrm{J} 17303$ is an outlier in period-spin space. On the other hand, the narrow-band NUSTAR 3-10 keV data fit with a 1T-PC model yields an effective ionization temperature of $k T_{\text {ion }}=10.2_{-1.1}^{+3.5} \mathrm{keV}$. For the RFL model, the observation angle was left as a free parameter, since there is no independent constraint. The 1T-RFL model yields $\chi_{\nu}^{2}=1.03$ with $k T=41_{-2}^{+3}$ $\mathrm{keV}$, However, the best-fit value of $r e l_{\text {refl }}$, which roughly characterizes the fraction of the downward incident radiation reflected by the WD surface, is greater than unity. To constrain this unphysical value, the results in Table 8 are shown for a value of $r e l_{\text {refl }}$ frozen to one.

Similar to the $1 \mathrm{~T}$ model fits, a PC model component is required to fit a $2 \mathrm{~T}$ model to the 3-50 NUSTAR spectra. Fitting a 2T-PC model yields $k T_{\text {low }}=14_{-10}^{+6} \mathrm{keV}$ and $k T_{\text {high }}=43 \pm 12 \mathrm{keV}$ (lower left panel in Figure 4) and we find that the 2T models are rather insensitive to the low-temperature value. We note that the measured $k T_{\text {low }}$ is consistent with the mean ionization temperature 
Table 3

Individual IP Comparison of Temperature and WD Mass Between Instruments

\begin{tabular}{|c|c|c|c|c|c|c|c|}
\hline \multirow[b]{2}{*}{ Instrument } & \multirow[b]{2}{*}{ Model } & \multirow[b]{2}{*}{$\begin{array}{c}\text { Energy Band } \\
\text { keV }\end{array}$} & \multicolumn{2}{|c|}{ TV Col } & \multicolumn{2}{|c|}{$\mathrm{J} 17303$} & \multirow[b]{2}{*}{ References } \\
\hline & & & $\begin{array}{c}\text { Temperature } \\
\text { keV }\end{array}$ & $\begin{array}{c}\text { Mass } \\
M_{\odot}\end{array}$ & $\begin{array}{c}\text { Temperature } \\
\text { keV }\end{array}$ & $\begin{array}{c}\text { Mass } \\
M_{\odot}\end{array}$ & \\
\hline$A S C A$ & $1 \mathrm{~T}$ & $5-10$ & $18_{-6}^{+14}$ & $\cdots$ & $\cdots$ & $\ldots$ & Ezuka \& Ishida (1999) \\
\hline Swift & $1 \mathrm{~T}$ & $14-195$ & $21.6 \pm 2.4$ & $\ldots$ & $37.1 \pm 4.4$ & $\ldots$ & Brunschweiger et al. (2009) \\
\hline INTEGRAL & $1 \mathrm{~T}$ & $20-100$ & $\ldots$ & $\ldots$ & $31.6_{-7.8}^{+12.7}$ & $\ldots$ & Landi et al. (2009) \\
\hline NUSTAR & IPM & $15-50$ & $\ldots$ & $0.77 \pm 0.03$ & $\ldots$ & $1.16 \pm 0.05$ & This work \\
\hline NuSTAR & IPM-PC/RFL ${ }^{a}$ & $3-50$ & $\cdots$ & $0.79 \pm 0.05$ & $\cdots$ & $1.1_{-0.1}^{+0.2}$ & This work \\
\hline Swift & IPM & $14-195$ & $\cdots$ & $0.78 \pm 0.06$ & $\cdots$ & $1.08 \pm 0.07$ & Brunschweiger et al. (2009) \\
\hline$A S C A$ & $\mathrm{Fe}$ line ratio ${ }^{\mathrm{c}}$ & $5-10$ & $\cdots$ & $0.51_{-0.22}^{+0.41}$ & $\cdots$ & $\cdots$ & Ezuka \& Ishida (1999) \\
\hline Suzaku & IP-PSR-PC ${ }^{\mathrm{d}}$ & $2-40$ & $\ldots$ & $0.91_{-0.10}^{+0.14}$ & $\ldots$ & $1.06_{-0.14}^{+0.19}$ & Yuasa et al. (2010) \\
\hline Suzaku & IP-PSR $^{\mathrm{d}}$ & $15-40$ & $\ldots$ & $0.87_{-0.18}^{+0.53}$ & $\ldots$ & $1.20_{-0.22}^{+0.15}$ & Yuasa (2013) \\
\hline
\end{tabular}

Notes. NUSTAR model descriptions can be found in Table 2 and in Section 3. The IPM model is based on the Suleimanov et al. (2005) mass model.

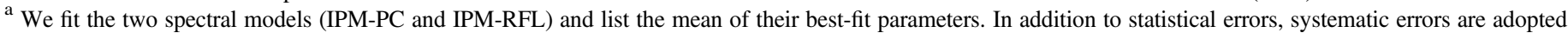
from the differences in the best-fit parameters between the two models.

b The higher temperature values are listed here.

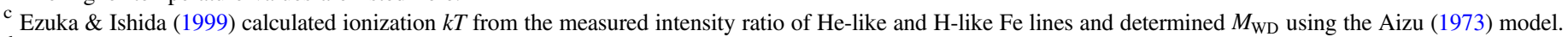

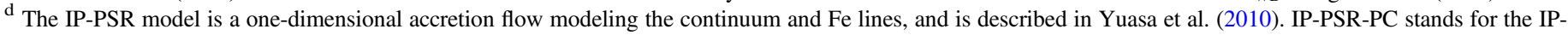
PSR model with partial-covering absorption.

of several field IPs measured by Ezuka \& Ishida (1999), $k T_{\text {ion }}=8.1 \mathrm{keV}$, as well as the $k T_{\text {low }} \approx 5 \mathrm{keV}$ from our simulation in Section 3.1.

\subsubsection{IPM Model Fitting}

While fitting an uncovered IPM model in the $3-50 \mathrm{keV}$ band gives an unacceptably high $\chi_{\nu}^{2}=3.9$, it fits well to the $15-50$ $\mathrm{keV}$ NUSTAR data giving a WD mass measurement of $M_{\mathrm{WD}}=1.16 \pm 0.05 M_{\odot}$ with $\chi_{\nu}^{2}=1.02$. The IPM-PC gives an excellent $\chi_{\nu}^{2}=1.00$ and a tightly constrained WD mass of $M_{\mathrm{WD}}=0.98 \pm 0.03 M_{\odot}$ (lower right panel in Figure 4). The partial-covering column density and fraction are in reasonable agreement with those seen in the 1T model. The IPM-RFL model gives a good $\chi_{\nu}^{2}=1.04$, ignoring the energy range $5.5-7.5 \mathrm{keV}$, but has a best-fit value of $r e l_{\text {refl }}$ greater than unity. With this value is frozen to one, the IPM model indicates an extreme WD mass of $M_{\mathrm{WD}}=1.34 \pm 0.02 M_{\odot}$.

The unphysical value of $r e l_{\text {refl }}$ obtained above motivates a hybrid model with both reflection and a partial covering, as shown in Table 8. This model gives sensible results for $r e l_{\text {ref } 1}$, observation angle, abundances, and covering fraction when IPM is used as the source spectrum, and yields a lower WD mass of $M_{\mathrm{WD}}=1.05_{-0.02}^{+0.03} M_{\odot}$. These masses are in much better agreement with those seen using only the partialcovering component. The lower partial-covering column density and covering fraction in the hybrid model demonstrate that the surface reflection has much the same effect as an accretion curtain. It is possible that further analysis of phaseresolved data will allow for better assessment of the relative contributions of intrinsic material and reflection to the emission spectrum. However for this phase-averaged data, using a PC model alone provides good fits to the spectrum.

\section{SYSTEMATICS ASSOCIATED WITH WD MASS AND TEMPERATURE MEASUREMENTS}

The temperatures and masses for TV Col and J17303 found in our NUSTAR analysis are compared with those found by other missions in Table 3. When similar spectral models are used, the NUSTAR results are consistent with these previous observations. A comparison of the various models, however, indicates that WD mass and temperature estimates have systematic uncertainties, largely due to the limited bandwidth (Section 5.1) and the effects of X-ray reprocessing components (Section 5.2), that are generally larger than the statistical uncertainties quoted by previous analyses.

\subsection{The Narrow 3-10 keV Band Spectral Fitting and Ionization Temperature}

As described in Section 3.1, we use the narrow energy band of 3-10 keV and a partially covered $1 \mathrm{~T}$ model to extract our effective ionization temperature. NUSTAR $3-10 \mathrm{keV}$ data fit by a 1T-PC model produces a mean ionization temperature $k T_{\text {ion }}=9.8 \pm 0.9(\mathrm{TV} \mathrm{Col})$ and $10.2_{-1.1}^{+3.5} \mathrm{keV}(\mathrm{J} 17303)$. For comparison, using the Fe line ratios measured by ASCA data, Ezuka \& Ishida (1999) measured $k T_{\text {ion }}=7.7 \pm 2.7 \mathrm{keV}$ for TV Col. However, the ionization temperature derived from soft $\mathrm{X}$-ray band fitting or Fe line ratios is not a reliable WD mass estimate for several reasons.

Since soft X-ray emission originates from near the bottom of the accretion column, the single ionization temperature does not represent the continuum (shock) temperature which is proportional to the WD mass. If the ionization temperature can be misinterpreted as the continuum temperature of the accretion column, the implied WD mass is grossly underestimated, thus it requires an additional model-dependent step to derive WD mass. 
Ezuka \& Ishida (1999) used the model relation developed by Aizu (1973) to convert $T_{\text {ion }}$ to $M_{\mathrm{WD}}$. However, this derivation can produce large errors associated with $M_{\mathrm{WD}}$ due to the large $d M_{\mathrm{WD}} / d T_{\text {ion }}$ gradient in the model for the relevant temperature range as well as the unknown temperature at the base of the accretion column $\left(k T_{\mathrm{B}}\right)$. Using the Aizu model with $k T_{\mathrm{B}}=0-2$ $\mathrm{keV}$, our ionization temperature for TV Col implies a WD mass of $M_{\mathrm{WD}}=0.8 \pm 0.1 M_{\odot}$ which is consistent with that determined from the broadband $3-50 \mathrm{keV}$ continuum fits, whereas a lower mass of $M_{\mathrm{WD}}=0.51_{-0.22}^{+0.42} M_{\odot}$ was derived from Ezuka \& Ishida (1999). The extreme sensitivity to $k T_{\text {ion }}$ measurement is evident as only a small $k T_{\text {ion }} \approx 2 \mathrm{keV}$ difference between the NUSTAR and ASCA results leads to WD mass discrepancy as large as $\Delta M_{\mathrm{WD}} \approx 0.3 M_{\odot}$. This sensitivity issue similarly affects $N U S T A R$ 3-10 keV spectral fitting for J17303 where $k T_{\text {ion }}=10.2_{-1.1}^{+3.5} \mathrm{keV}$ leads to $M_{\mathrm{WD}}=0.8_{-0.1}^{+0.6} M_{\odot}$, while the broadband spectral fit with the IPM models yield a higher WD mass $M_{\mathrm{WD}}=1.16 \pm 0.12 M_{\odot}$ with significantly smaller errors.

Overall, the mean WD mass measured in Ezuka \& Ishida (1999) is systematically underestimated by $\sim 0.3 M_{\odot}$, compared to the broadband fit. The mean ionization temperature of the 13 field IPs was measured by Ezuka \& Ishida (1999) to be $k T \approx 8.8 \mathrm{keV}$. This corresponds to a mean WD mass of $0.55 M_{\odot}$, which is considerably less than the mean field IP mass of $0.86 \pm 0.07 M_{\odot}$ measured by INTEGRAL and XMM-Newton (Bernardini et al. 2012) and the mean mass of the 24 IPs measured by Yuasa et al. (2010), $0.88 \pm 0.25 M_{\odot}$. The narrow energy band of $A S C A$, with its sharply decreasing effective area above $7 \mathrm{keV}$, makes it difficult to accurately estimate the soft $\mathrm{X}$-ray continuum, leading to a large uncertainty in the temperature and a best-fit WD mass that is much lower than other observation.

Thus we conclude that soft X-ray (narrow) band fits are an unreliable WD mass estimator. The narrow-band fits underestimate ionization temperature, leading to systematically lower WD masses, or masses with very large errors. This conclusion is clear for the ASCA results on IP masses compared to NUSTAR and XMM-Newton/INTEGRAL. This conclusion will also pertain to narrow-band temperature measurements with Chandra or XMM-Newton. But XMM-Newton plus INTEGRAL results, broadband Suzaku results, and broadband NuSTAR results all produce good agreement on WD masses.

\subsection{WD Mass Measurements}

It is evident that including a PC or RFL model systematically decreases the measured temperature and WD mass for TV Col and J17303. The temperature and WD mass are very sensitive to the partial-covering column density, thus they can be largely overestimated if the X-ray reprocessing is significant but it is not taken into account for spectral fitting. Therefore, the most physically well motivated model, an IPM model with partial covering and/or reflection, that is fit over a broad energy band provides the most robust mass estimate. Although the IPM-PC and IPM-RFL models show nearly identical spectral shapes thus yielding similar fit quality, they give small but finite differences in the fit WD mass values. Then, we take a range of the best-fit WD masses by different spectral models as systematic errors. As shown in Table 3, the systematic errors due to applying different models dominate over the statistical errors.

For TV Col, the IPM model fits yield $M_{\mathrm{WD}}=0.79 \pm 0.05 M_{\odot}$, where the systematic errors corresponding to the difference in the best-fit $M_{\mathrm{WD}}$ values between the IPM-PC and IPM-RFL models are added to the statistical errors. For J17303, the 3-50 keV band fits with the three IPM models (the IPM-PC, IPM-RFL with re $l_{\text {ref } 1}$ fixed to 1, and IPM-PC-RFL models) that are statistically acceptable and physically reasonable give the mean WD mass of $M_{\mathrm{WD}}=1.1_{-0.1}^{+0.2} M_{\odot}$. Given that our results are in good agreement with the Suzaku results (Yuasa 2013) where the Fe lines were fit between 6 and $7 \mathrm{keV}$, the NUSTAR observations establish that broadband $3-50 \mathrm{keV}$ observations are effective in constraining temperatures and masses without the need to fit the $5.5-7.5 \mathrm{keV}$ soft X-ray line emission region.

On the other hand, fitting only the $E>15 \mathrm{keV}$ spectrum yields a WD mass, $M_{\mathrm{WD}}=0.77 \pm 0.05 \quad(\mathrm{TV} \mathrm{Col})$ and $1.16 \pm 0.05 M_{\odot}(\mathrm{J} 17303)$, consistent with the broadband fit to an IPM model with partial covering or reflection. Although the limited bandpass induces larger statistical errors, this approach gives another robust measurement of WD mass as well as $k T_{\text {high }}$ in the $2 \mathrm{~T}$ model since it removes the dependence on partial covering and/or reflection which are neglibile at $E>15 \mathrm{keV}$. This is further supported by the results of fitting our simulated partially covered IPM model with a range of IP mass $M_{\mathrm{WD}}=0.8-1.0 M_{\odot} \quad$ and column density up to $N_{H}^{\mathrm{pc}} \sim 3 \times 10^{24} \mathrm{~cm}^{-2}$ with an uncovered IPM model at high energies, which returns the correct WD mass. Based on our simulation and NUSTAR data fitting, we find that the $E>15 \mathrm{keV}$ band for the two IPs is optimal since partial covering does not affect the WD mass measurement and statistical errors remain small.

For both TV Col and J17303, we obtain the consistent WD masses between the two fitting methods, and they are in good agreement with the previous measurements (Table 3). Similarly, using a large sample of field IPs and their Suzaku data, Yuasa (2013) demonstrated that broadband spectral fits with a partially covered IPM model and high-energy $(E>15 \mathrm{keV})$ fits with an uncovered IPM model yield essentially the same WD masses within statistical uncertainties. Our results for TV Col and J17303 are consistent with the INTEGRAL and Swift analysis extending to $E \gtrsim 100 \mathrm{keV}$ (Brunschweiger et al. 2009; Landi et al. 2009), thus our 3-50 keV band with NuSTAR is sufficiently "broad" to measure WD masses accurately. In summary, a combination of the two fitting methods with NuSTAR broadband X-ray spectroscopy yields the most accurate measurement of WD masses.

\section{SPECTRAL ANALYSIS OF THE CHXE}

The results of our analysis of TV Col and J17303 lead us to conclude that narrow-band low-energy measurements can underestimate WD masses, whereas high-energy measurements of the continuum temperature lead to robust mass estimates. Thus, a re-evaluation of the origin of the CHXE and its relation to the point source populations in the larger GC region is warranted, and we perform a reanalysis of XMM-Newton and NUSTAR data from the CHXE using the spectral models discussed above.

\subsection{Thermal Plasma Model Fitting}

In order to compare our measurements of the CHXE with the soft X-ray measurements of the larger GC region, we fit only the 2-10 keV XMM-Newton spectrum of the southwest CHXE region (as defined in KP15) with the same $2 \mathrm{~T}$ model with no partial covering that was used in previous Chandra (Muno 
Table 4

GC Plasma Temperature Comparison Between this Work and Previous Measurements

\begin{tabular}{|c|c|c|c|c|c|c|}
\hline Region or Source & X-ray Telescope & Energy Band (keV) & Models & $k T_{2}(\mathrm{keV})$ & $k T_{3}(\mathrm{keV})$ & References \\
\hline SW region in CHXE & XMM-Newton only & $2-10$ & $2 \mathrm{~T}$ & $7.7_{-1.0}^{+0.8}$ & $\ldots$ & This work \\
\hline just outside CHXE & XMM-Newton & $2-10$ & $2 \mathrm{~T}^{\mathrm{a}}$ & 7.5 & $\cdots$ & HW13 \\
\hline SW region in $\mathrm{CHXE}$ & NUSTAR & $2-40$ & $3 \mathrm{~T}$ & $7.2_{-1.3}^{+1.4}$ & $58_{-23}^{+127}$ & KP15 \\
\hline $2^{\circ} \times 0.8 \mathrm{GC}$ field & Chandra & $2-10$ & $2 \mathrm{~T}^{\mathrm{a}}$ & $\gtrsim 8$ & $\cdots$ & Muno et al. (2004a) \\
\hline
\end{tabular}

Note. All models presented in this table fit two or three thermal components. The lowest temperature component fits to $k T_{1} \sim 1 \mathrm{keV}$ diffuse emission, and it is not listed here. $k T_{2}$ refers the higher temperature of the $2 \mathrm{~T}$ models or the middle temperature of the $3 \mathrm{~T}$ models. $k T_{3}$ refers to the highest temperature of the $3 \mathrm{~T}$ models. ${ }^{a}$ HW13 fit the thermal bremsstrahlung model, while the XSPEC model mekal (Mewe et al. 1986) was used by Muno et al. (2004a). In the both cases, the lower temperature component in their two-temperature models represent $k T \sim 1$ thermal diffuse emission.
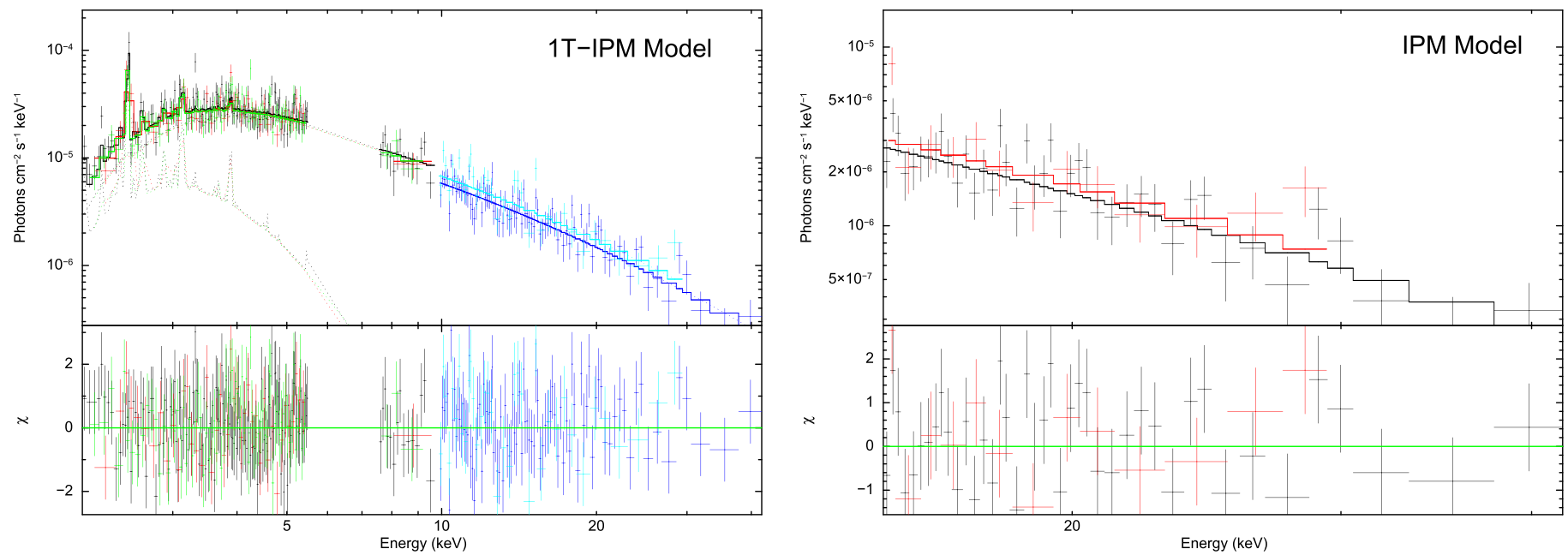

Figure 2. XMM-Newton + NuSTAR 2-40 keV unfolded spectra of the CHXE southwest region (XMM-Newton below $10 \mathrm{keV}$, NuSTAR above $10 \mathrm{keV}$ ) fit with the $1 \mathrm{~T}$ IPM (lower panel, without 5.5-7.5 keV band) and IPM models (right panel, above $15 \mathrm{keV}$ ).

et al. 2004a) and XMM-Newton (HW13) studies of the surrounding GC region. In these previous analyses, the lower temperature accounts for a diffuse plasma with temperature $k T_{1} \approx 1 \mathrm{keV}$ and the higher temperature of $k T_{2} \approx 7-8 \mathrm{keV}$ is interpreted as arising from a population of magnetic $\mathrm{CVs}$ with mean WD mass $M_{\mathrm{WD}} \approx 0.5 M_{\odot}$. In our analysis, we recover a $k T_{2}=7.7_{-1.0}^{+0.8} \mathrm{keV}$ that is consistent with these previous works. See Table 4 listing a comparison of the temperatures of the GC diffuse emission derived from the various models applied in different energy bands. The abundance for the high-temperature component, presumably associated with accretion onto the WD, is approximately solar, and the column density is consistent with previous GC measurements.

Using broad 2-40 keV energy-band data, KP15 fit the joint XMM-Newton and NuSTAR spectrum of the southwest CHXE region to a $3 \mathrm{~T}$ model with no partial covering, yielding $k T_{1}=1.0_{-0.4}^{+0.3}, k T_{2}=7.5_{-1.3}^{+1.6}$ and $k T_{3}=58_{-23}^{+127} \mathrm{keV}$. In this model, one temperature represents the diffuse, cool plasma, and the other two represent the standard two-temperature component used for TV Col and J17303 above. The first $\left(k T_{1}=1.0 \mathrm{keV}\right)$ and second temperatures $\left(k T_{2}=7.5 \mathrm{keV}\right)$ agree with the $X M M$-Newton only measurement as well as Muno et al. (2004a) and HW13. The third bremsstrahlung component, largely fitting the hard X-ray continuum, sets a lower limit of $k T>35 \mathrm{keV}$ (KP15).
Table 5

Joint XMM-Newton and NUSTAR Spectral Fitting Results for the CHXE Southwest Region

\begin{tabular}{lcc}
\hline \hline Parameters & 1T-IPM & IPM \\
\hline Instrument & XMM + NuSTAR & NuSTAR \\
Bandpass $[\mathrm{keV}]$ & $2-40$ & $15-40$ \\
$N_{\mathrm{H}}^{f}\left[10^{22} \mathrm{~cm}^{-2}\right]$ & $14 \pm 1$ & $\ldots$ \\
$k T_{1}[\mathrm{keV}]$ & $1.0 \pm 0.2$ & $\ldots$ \\
$Z_{1}$ & $5.0_{-3.2}$ & $\ldots$ \\
$N_{1}\left[10^{-2} \mathrm{~cm}^{-3}\right]$ & $8.43_{-0.02}^{+0.21}$ & $\ldots$ \\
$M_{\mathrm{WD}}\left[M_{\odot}\right]$ & $0.87_{-0.09}^{+0.11}$ & $0.89_{-0.12}^{+0.15}$ \\
$N_{\text {IPM }}\left[10^{-13}\right]$ & $4.9_{-1.3}^{+1.7}$ & $4.8_{-1.7}^{+2.5}$ \\
Flux $(2-10 \mathrm{keV})^{\mathrm{a}}$ & 1.2 & 1.2 \\
Flux $(20-40 \mathrm{keV})^{\mathrm{a}}$ & 0.7 & 0.7 \\
$\chi_{\nu}^{2}(\mathrm{dof})$ & $1.07(400)$ & $1.12(52)$ \\
\hline
\end{tabular}

Note. $k T_{1}$ corresponds to the $1 \mathrm{keV}$ thermal thermal diffuse emission in the GC and is unrelated to the IPs.

${ }^{a}$ The flux unit is $10^{-12} \mathrm{erg} \mathrm{cm}^{-2} \mathrm{~s}^{-1}$.

\subsection{IPM Model Fitting}

Following the analysis for TV Col and J17303, we fit two additional spectral models to the CHXE data (Figure 2 and Table 5). The 2-40 keV joint XMM-Newton and NuSTAR spectra of the southwest CHXE region fit to a $k T_{1} \sim 1 \mathrm{keV}$ APEC plus IPM model (1T-IPM), with the 5.5-7.5 keV region 
excluded and no partial covering, results in $\chi_{\nu}^{2}=1.07$ and a mean WD mass of $M_{\mathrm{WD}}=0.87_{-0.09}^{+0.11} M_{\odot}$. The abundance of the cool plasma is poorly constrained, but consistent with previous measurements of the GC region. We also fit the $\sim 1 \mathrm{keV} 1 \mathrm{~T}$ IPM-PC model, as we argue that the most physical model is the IPM model with partial covering for the case of TV Col and J17303. While the fit was acceptable with $\chi_{\nu}^{2} \approx 1$, the partialcovering column density for the CHXE region is poorly constrained, due to spectral contamination from the diffuse $k T \approx 1 \mathrm{keV}$ plasma and the interstellar absorption in the region.

To check whether the model without partial covering is accurately measuring the mean WD mass, we performed a fit using NUSTAR 15-40 keV data. For both TV Col and J17303, fits to the $E>15 \mathrm{keV}$ X-ray continuum yield reliable masses and hard X-ray continuum temperatures that are consistent with both NUSTAR broadband fits and measurements by other observatories (see Tables 3, 7, and 8). At these high energies, a partial covering does not affect the measured temperature. The 15-40 keV NuSTAR fit to the CHXE southwest region yields a mean WD mass of $M_{\mathrm{WD}}=0.89_{-0.12}^{+0.15} M_{\odot}$ (see Table 5), in agreement with the $2-40 \mathrm{keV}$ joint XMM-Newton and NuSTAR fit with the 1T-IPM model. Similarly, we fit an uncovered IPM model to the 15-40 keV NUSTAR data of the northeast region of the CHXE (as defined in KP15). The best-fit WD mass from the northeast region is $M_{\mathrm{WD}}=0.95_{-0.12}^{+0.14} M_{\odot}$ and is consistent with that of the southwest region. However, the northeast region is contaminated by a $k T \sim 5 \mathrm{keV}$ thermal component from Sgr A East and non-thermal emission from several prominent X-ray filaments. Based on these considerations, we conclude that the most reliable mass estimate for the CHXE is obtained by fitting the southwest spectrum above $15 \mathrm{keV}$ to an IPM model without partial covering, yielding a mass of $M_{\mathrm{WD}}=0.89_{-0.12}^{+0.15} M_{\odot}$.

\section{DISCUSSION}

\subsection{The Origin of the CHXE}

In light of our analysis of TV Col, J17303, and the CHXE using a wide variety of IP spectral models, we propose that the $\mathrm{CHXE}$ is an unresolved population of IPs with mean WD mass $M_{\mathrm{WD}} \approx 0.9 M_{\odot}$. We also list other observational evidence supporting the IP scenario.

1. In the soft X-ray band, the southwest region of the CHXE outside the supernova remnant Sgr A East shows $k T \approx 8 \mathrm{keV}$ thermal emission with both neutral and ionized $\mathrm{Fe}$ lines in addition to the diffuse $k T \sim 1 \mathrm{keV}$ component, indicating a dominant population of CVs. While millisecond pulsars, LMXBs, or non-thermal diffuse emission can account for the CHXE as a powerlaw spectrum with $\Gamma \sim 1.5-2$ above $20 \mathrm{keV}$, their lowenergy extensions below $10 \mathrm{keV}$ are inconsistent with the observed thermal emission with Fe lines.

2. The CHXE exhibits an elliptical profile similar to the surface brightness distribution of the nuclear star cluster (NSC) where a high stellar density is observed in the IR band with a total luminosity of $\sim 10^{7} L_{\odot}$ and mass of $\sim 10^{7} M_{\odot}$. In contrast to previous IR studies, after correcting the effects of polycyclic aromatic hydrocarbon emission and extinction carefully, Schödel et al. (2014) revealed a NSC profile flattened along the Galactic plane and centered at $\mathrm{Sgr} \mathrm{A}^{*}$ with a half light radius of $4.2 \pm$ $0.4 \mathrm{pc}$. The similarity between the two spatial distributions strongly suggests a stellar origin for the CHXE.

3. The CHXE has spectral characteristics closely matching those of the field IPs observed by both INTEGRAL/ XMM-Newton (Bernardini et al. 2012) and Suzaku (Yuasa et al. 2010), including the derived masses. On the other hand, both non-magnetic CVs and polars have too soft $\mathrm{X}$-ray spectra to account for the $20-40 \mathrm{keV}$ emission of the CHXE (Cropper et al. 1998; Byckling et al. 2010). The INTEGRAL/IBIS Galactic Plane survey detected one non-magnetic CV (SS Cyg) and three polars, while 60\% of the known IPs were detected (Barlow et al. 2006).

4. The mean WD mass of the $\operatorname{CHXE}\left(M_{\mathrm{WD}} \approx 0.9 M_{\odot}\right)$ is remarkably consistent with that of the field IPs $\left(M_{\mathrm{WD}}=0.86-0.88 M_{\odot}\right.$; Bernardini et al. 2012; Yuasa et al. 2010). Also, the mean WD mass among all CVs measured by SDSS is $M_{\mathrm{WD}}=0.83 \pm 0.24 M_{\odot}$ (Zorotovic et al. 2011) in excellent agreement with the $M_{\mathrm{WD}} \approx 0.9 M_{\odot}$ derived for the $\mathrm{CHXE}$ (Table 6).

We thus conclude that the CHXE is consistent with one population of IPs with $M_{\mathrm{WD}} \approx 0.9 M_{\odot}$. The dominant $k T \approx 8 \mathrm{keV}$ component detected in the soft X-ray band corresponds to the lower temperature $\left(k T_{\text {low }}\right)$ present in $2 \mathrm{~T}$ model fits to one-dimensional accretion flow models of IPs with $M_{\mathrm{WD}} \approx 0.9 M_{\odot}$, where it is associated with the emission near the base of the accretion column or with the ionization temperature in the line emission region.

The lower mass $M_{\mathrm{WD}} \approx 0.5 M_{\odot}$ obtained from Chandra and XMM-Newton data (Muno et al. 2004b, 2004a; Heard \& Warwick 2013) can be explained as an artifact of limited, low energy-band spectral fitting. As we demonstrated in Section 5.1, the narrow-band soft X-ray measurements underestimate the IP masses, and a precise measurement of the mean WD mass from the CHXE region (which is unresolved from the larger GC region by INTEGRAL and Suzaku) is only possible through NUSTAR.

Recently it has been suggested that highly magnetized CVs are formed in a common envelope phase (Tout et al. 2008). This has been posited to explain why high magnetic field WDs (HMFWDs) are more massive on average than the general WD population. The mean mass of the HMWFDs, $M_{\mathrm{WD}} \approx 0.8 M_{\odot}$ (Kawka et al. 2007; Ferrario et al. 2015), is strikingly similar to that measured for the IP population comprising the CHXE. Since the IPs are believed to represent an alternative endpoint of the common envelope phase, it may be natural that they are more massive as well. This makes them hotter and thus easier to detect in hard X-ray observations.

\subsection{Luminosity Function and Density of IPs in the GC}

We investigate the luminosity function of the IPs in the CHXE. We use the total $2-8 \mathrm{keV}$ luminosity $L_{X}=3.6 \times 10^{34} \mathrm{erg} \mathrm{s}^{-1}$ in the CHXE excluding the $k T \sim 1 \mathrm{keV}$ diffuse component (KP15), a power-law index for the luminosity distribution $\left(N\left(>L_{X}\right)=k L_{X}^{-\alpha}\right)$ with $\alpha \sim 1-1.5$ (Muno et al. 2009; Yuasa et al. 2012) and an $L_{X}^{\max } \sim 3 \times 10^{33} \mathrm{erg} \mathrm{s}^{-1}$ for the maximum IP luminosity (Pretorius \& Mukai 2014). The results are quite insensitive to the exact $L_{X}^{\max }$, provided $L_{X}^{\min } / L_{X}^{\max } \ll 1$. An upper bound on $L_{X}^{\min }$ is obtained from the observation that the CHXE point sources are completely unresolved by $N u S T A R$. Given the 
NUSTAR angular resolution of $\sim 1 \mathrm{pc}$ at the GC, this requires at least few hundred sources. This requires an X-ray luminosity function (XLF) extending down to $L_{X} \lesssim 5 \times 10^{31} \mathrm{erg} \mathrm{s}^{-1}$. The corresponding $\log N-\log L$ would suggest that the $\mathrm{CHXE}$ is composed primarily of the Chandra point sources in the $N U S T A R$ field of view. An estimation of the IP density in the GC also suggests that the XLF of the CHXE IPs has $L_{\min } \lesssim 5 \times 10^{31} \mathrm{erg} \mathrm{s}^{-1}$.

We can estimate the IP density directly from the density of main sequence stars in the GC. From Pretorius et al. (2013), we find that the central $10 \mathrm{pc}$ contains $\sim 7500$ main sequence stars per $\mathrm{pc}^{3}$. The IP density is $n(\mathrm{IP})=7500 * f_{\mathrm{ab}} * f_{\mathrm{B}} *$ $f_{\mathrm{bin}} * f_{\mathrm{cv}} * f_{\mathrm{ip}} \mathrm{pc}^{-3}$. Here $f_{\mathrm{ab}} \sim 0.28$ is the fraction of main sequence stars with mass $\sim 4.5-10 M_{\odot}$ (A and B stars) sufficient to form $M_{\mathrm{WD}}>0.8 M_{\odot}$ (Ferrario et al. 2005). The fraction of $\mathrm{A}$ and $\mathrm{B}$ stars that will form highly magnetized WDs is estimated as $f_{\mathrm{B}} \sim 0.4$ (Ferrario et al. 2005). A number $\sim 2$ times smaller still can be consistent with the estimated number of isolated, highly magnetized WDs presented in Ferrario et al. (2005). Hopman (2009) estimated the binary fraction $f_{\text {bin }}$ as a function of normalized radius from the GC. Inside the influence radius, which is the radius within which the supermassive black hole dominates dynamical processes, the binary fraction drops sharply. Outside the influence radius, Hopman (2009) quotes a binary fraction $f_{\text {bin }} \sim 0.05$. The influence radius is of the order of a few pc, so the fraction of the total mass at $r<10 \mathrm{pc}$ inside the influence radius is small, and so we estimate the binary fraction as $f_{\text {bin }} \sim 0.05$. We very crudely set the fraction of WD binaries that are $\mathrm{CVs}\left(f_{\mathrm{cv}}\right)$ to $\sim 1$, but this is a crude upper limit, since only WDs in tight binaries will form CVs. The fraction of magnetic CVs among all CVs is $f_{\text {mcv }} \sim 0.2$ (Pretorius et al. 2013). Based on a sample of 30 magnetic CVs detected by the ROSAT Bright Survey, Pretorius et al. (2013) estimated $f_{\text {ip }} \sim 0.4$ as the fraction of IPs among the magnetic CVs in the solar neighborhood. This yields $n_{\mathrm{ip}} \approx 2-4 \mathrm{pc}^{-3}$ where the lower density assumes $f_{\mathrm{B}} \sim 0.2$.

These IP densities are in good agreement with the estimate of HW13, $n_{\text {ip }} \sim 3-10 \mathrm{pc}^{-3}$, near the southwest region of the CHXE. KP15 estimated a higher IP density, $n_{\text {ip }} \sim 15 \mathrm{pc}^{-3}$, because there it was assumed that the XLF of IPs extended well below $10^{31} \mathrm{erg} \mathrm{s}^{-1}$. However, an XLF of the CHXE extending to $\lesssim 5 \times 10^{31} \mathrm{erg} \mathrm{s}^{-1}$ gives an IP density of $\gtrsim$ few $\mathrm{pc}^{-3}$, consistent with a NuSTAR unresolved CHXE point source population, the rough first principles estimate above and in HW13. It is tempting to regard these estimates as conservative since accretion rate calculations (Yuasa et al. 2012) would suggest that WD mass could have grown $\sim 0.05-0.1 M_{\odot}$ over several Gyr. Using an analytic initial (progenitor)-final (WD) mass function (Catalán et al. 2008), IPs with $M_{\mathrm{WD}} \sim 0.8-0.9 M_{\odot}$ would be $\sim 0.05-0.1 M_{\odot}$ lighter at formation, implying progenitor masses $\sim 0.8-1 M_{\odot}$ lighter. Thus a larger fraction of main sequence stars would be available to produce massive IPs. However the question of whether CVs actually gain mass by accretion or suffer mass loss through nova cycles is complex and unresolved (Wijnen et al. 2015).

Previous Chandra observations suggested that a large population of the X-ray point sources in the GC region are magnetic CVs, and most likely IPs. Muno et al. (2004a) classified a majority of thousands of X-ray point sources in the central $2^{\circ} \times 0.8$ region as possessing hard power-law photon indices $\Gamma \lesssim 1$ indicating that they are mostly IPs. Hong et al. (2012) found that a low extinction region called the "limiting window", at $\sim 1.4$ south of the GC, contains a large population of magnetic CVs.

Recently, an extensive NuSTAR survey detected 70 hard $\mathrm{X}$-ray point sources over a $0.6 \mathrm{deg}^{2}$ region in the GC region, and nearly all of them have Chandra counterparts (Hong et al. 2016). Their $10-40 \mathrm{keV} \log N-\log S$ distribution indicates that a majority of the NUSTAR hard X-ray sources should have $k T \sim 20-40 \mathrm{keV}$ to match the Chandra source distribution. More than a dozen bright NUSTAR sources exhibit broadband $\mathrm{X}$-ray spectra that resemble those of heavy IPs $\left(\sim 0.8-1 M_{\odot}\right)$ with Fe lines and $k T \sim 20-40 \mathrm{keV}$ when fit with a 1T APEC model. The NUSTAR point sources detected above $10 \mathrm{keV}$ represents the bright tail $\left(L_{X} \gtrsim 4 \times 10^{32} \mathrm{erg} \mathrm{s}^{-1}\right.$ in the $3-10 \mathrm{keV}$ band) of the Chandra source luminosity distribution or only $\sim 1 \%$ of the GC Chandra source population in the GC.

We can estimate the contribution of IPs to the point sources in the $2 \times 0.8 \mathrm{deg}^{2}$ region under the assumption that the CHXE XLF applies. The XLF is normalized by the $\gtrsim 35$ sources (all with Chandra counterparts) that Hong et al. (2016) observed and claimed to be IPs, and by the ratio of the areas surveyed (0.6 deg ${ }^{2}$ and $1.6 \mathrm{deg}^{2}$, respectively). If the XLFs of the CHXE and the Chandra sources are comparable, $L_{X}^{\min } \lesssim 5 \times 10^{31} \mathrm{erg} \mathrm{s}^{-1}$ implies that $\gtrsim 20 \%-40 \%$ of the Chandra sources are IPs. The range of our estimates is due to the uncertainty in the slope of $\log N-\log L$ curves. If the $N U S T A R$ sources observed by Hong et al. (2016) are primarily IPs and/or if the XLF of the CHXE IPs extends down by another factor of $\sim 2$ (which is close to the sensitivity limit of the Chandra GC survey), an overwhelming majority of the Chandra point sources in the GC could be IPs.

\subsection{IPs and the Galactic Ridge and Bulge Populations}

Observations of the Galactic ridge and bulge have also suggested a dominant population of IPs. Yuasa et al. (2012), using Suzaku data, fit the $E>15 \mathrm{keV}$ spectrum of the bulge with a one-dimensional accretion flow model. The derived mean mass was $M_{\mathrm{WD}}=0.66_{-0.07}^{+0.09} M_{\odot}$, which is inconsistent with the mean mass we estimate for the CHXE and field IPs (Table 6). A similar mean WD mass $\left(M_{\mathrm{WD}}=0.60 \pm 0.05 M_{\odot}\right)$ was obtained by Türler et al. (2010) using the INTEGRAL Galactic ridge data. The INTEGRAL and Suzaku IP model fits yield consistent results with masses $\sim 0.6-0.66 M_{\odot}$ even when the energy band is restricted to $E \gtrsim 15-20 \mathrm{keV}$, and as we demonstrated, such hard X-ray band fits should yield the most reliable WD mass measurements. Note that using broad energyband fits (3-50 keV) to the Suzaku ridge observations, the mean observed mass decreased to $\sim 0.48 M_{\odot}$, similar to the $0.5 M_{\odot}$ obtained from INTEGRAL observations of the Galactic ridge (Krivonos et al. 2007).

The lower mean IP mass measured for the Galactic ridge, compared to the GC, is puzzling. As mentioned above, the higher IP mass in the GC is more consistent with observations of CV masses in general and the HFMWDs, which are believed to share a common evolutionary origin with magnetic CVs. Given that both magnetic and non-magnetic CVs have the same high mass $\sim 0.8-0.9 M_{\odot}$, why would the IPs of the Galactic ridge have such markedly lower masses? One clue could be the systematically lower temperatures or softer power-law spectra observed in the Galactic ridge. This is also seen in the derived temperatures, with the CHXE being substantially hotter than the $k T \approx 15 \mathrm{keV}$ measured by Suzaku and INTEGRAL. Additionally, broadband RXTE (Valinia \& Marshall 1998) 

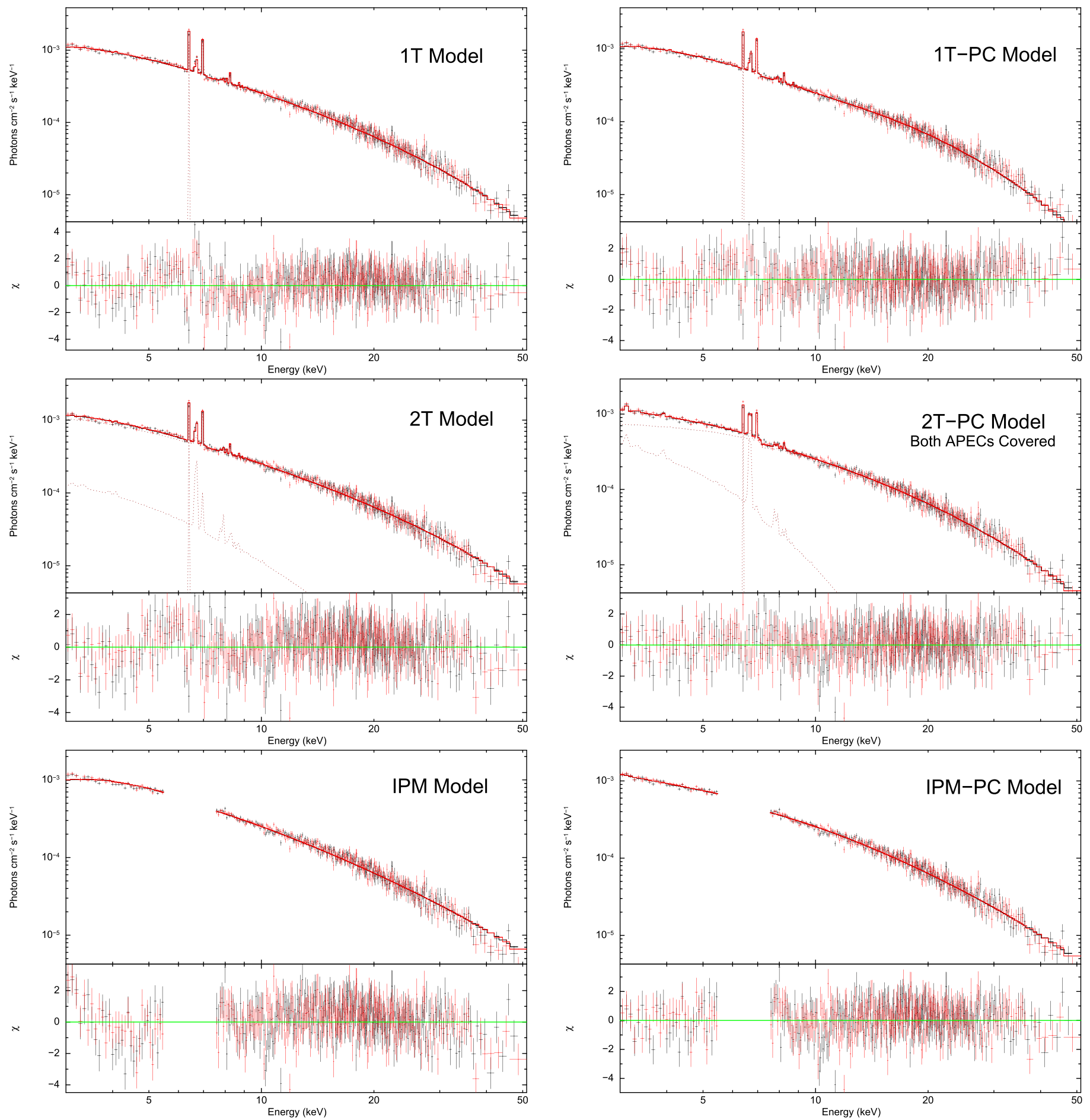

Figure 3. NuSTAR 3-50 keV unfolded spectra of TV Col (black: FPMA; red: FPMB) fit with 1T models (top panels), 2T models (middle panels), and IPM models by ignoring the Fe line complex in the 5.5-7.5 keV band (bottom panels). The left and right panels show the spectral fits without and with partial-covering absorption, respectively. The spectral fits with the RFL model are not presented here since they show similar residuals and fit quality as the PC models (Table 7).

and Suzaku (Yuasa et al. 2012) fits using a power-law model demonstrated softer photon indices $(\Gamma=2.3$ and $\Gamma=2.8$, respectively) than corresponding fits to the CHXE $(\Gamma=1.2-1.9 ; \mathrm{KP} 15)$.

What is required to account for the softer ridge emission is a dominant contribution from non-magnetic $\mathrm{CVs}$ and polars since their spectra are softer on average than IPs (Eracleous et al. 1991; Cropper et al. 1998; Byckling et al. 2010; Reis et al. 2013). If the IPs went from being the dominant CV component in the GC to a sub-dominant component, compared to non-magnetic $\mathrm{CVs}$ and polars, in the Galactic ridge, then a spectral softening would result. Applying the IP model, as Suzaku and INTEGRAL did, to a spectrum dominated by softer non-magnetic CVs and polars, would lead to a systematically lower WD mass. Indeed, fitting a single optically thin thermal plasma model to the X-ray spectra of local non-magnetic CVs 

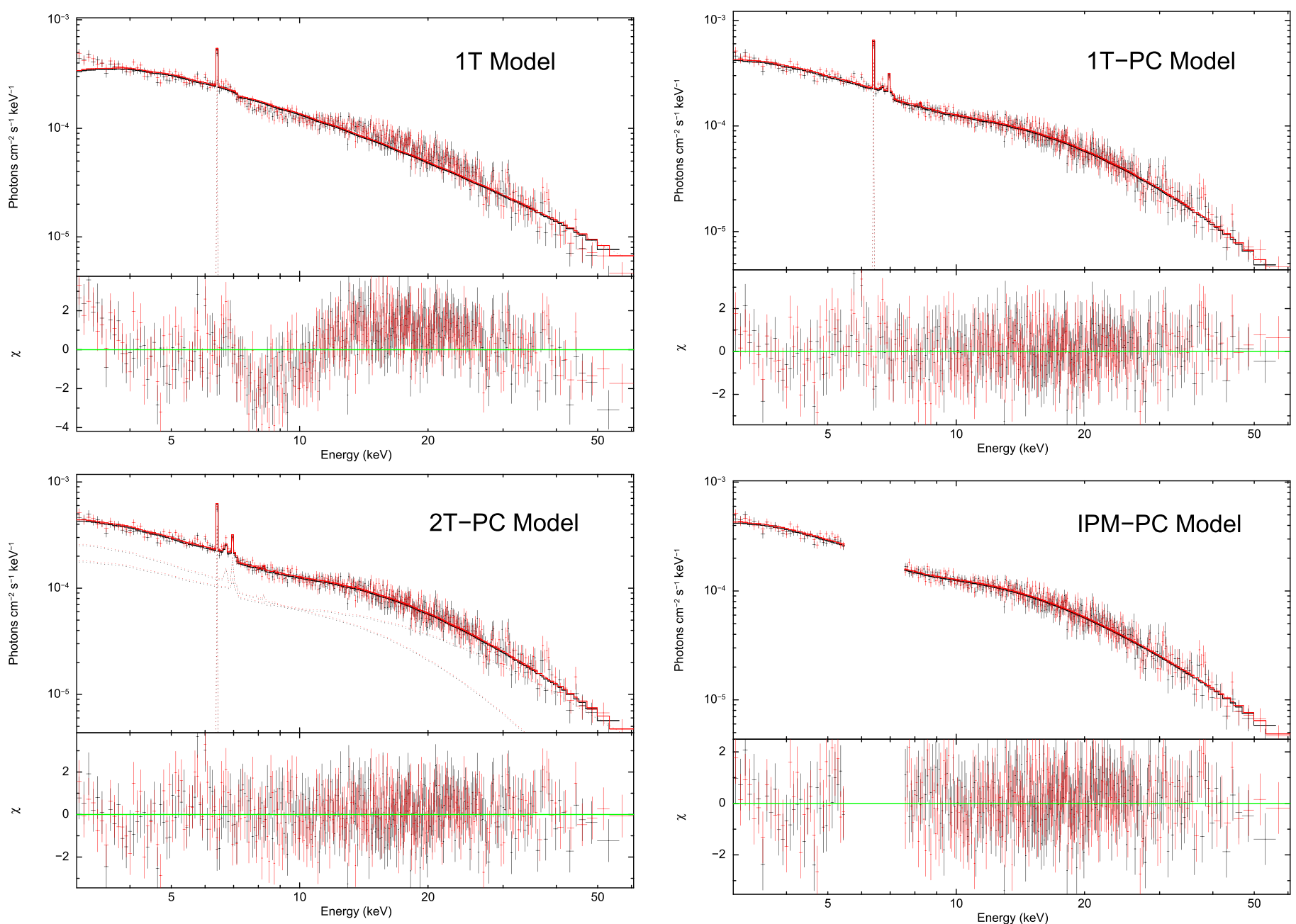

Figure 4. NuSTAR 3-50 keV unfolded spectra of J17303 (black: FPMA; red: FPMB) fit with 1T, 2T, and IPM models. A Compton reflection hump from reflection of $\mathrm{X}$-rays off the WD surface is clearly visible in the residuals above $20 \mathrm{keV}$ (top left panel). The spectral fits with the RFL model are not shown here since they exhibit similar residuals as in the PC models (see Table 8 for the fitting results with the RFL model).

Table 6

Mean WD Mass Comparison Between this Work and Previous Measurements

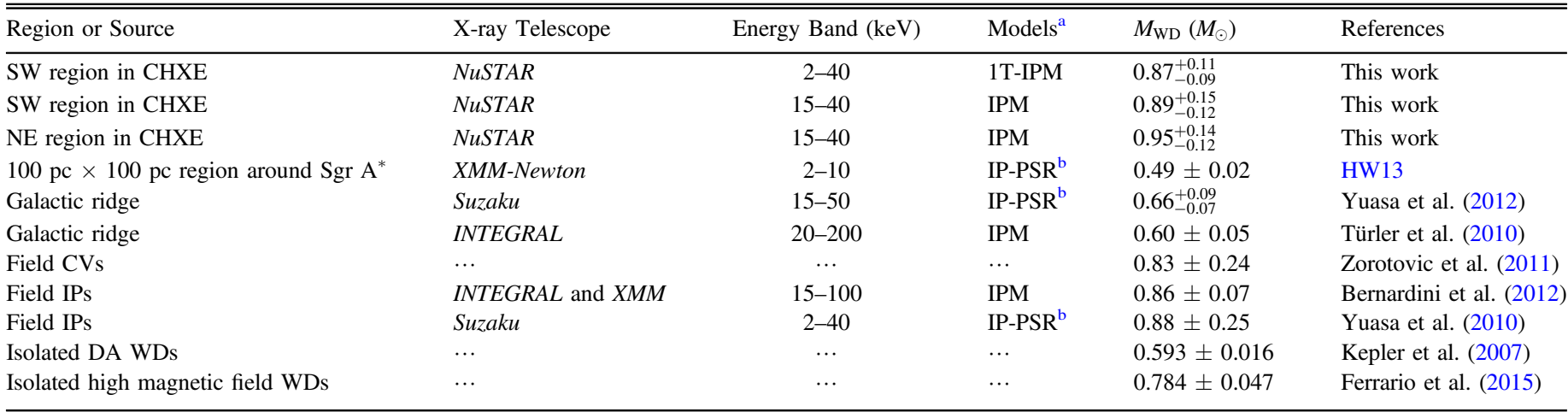

Notes. NUSTAR model descriptions can be found in Table 2 .

a $5.5-7.5 \mathrm{keV}$ data bins are ignored in NUSTAR IPM model fittings.

$\mathrm{b}$ The IP-PSR model is a one-dimensional accretion flow modeling the continuum and Fe lines, and is described in Yuasa et al. (2010).

yields $k T \lesssim 10 \mathrm{keV}$, which is significantly softer than TV Col $(k T \approx 20 \mathrm{keV}$ ) and most IPs (Byckling et al. 2010).

Recently, Reis et al. (2013) found an average X-ray luminosity of 20 optically selected non-magnetic CVs is $8 \times$
$10^{29} \mathrm{erg} \mathrm{s}^{-1}$, contrary to the previous studies whose samples were biased toward selecting CVs with higher accretion rates. This indicates that a large population of non-magnetic CVs may remain unresolved at a distance of $8 \mathrm{kpc}$ but they may 
Table 7

NuSTAR Spectral Fitting Results of TV Col

\begin{tabular}{|c|c|c|c|c|c|c|c|c|c|c|}
\hline Parameters & $1 \mathrm{~T}$ & 1T-PC & 1T-PC & 1T-RFL & $2 \mathrm{~T}$ & $2 \mathrm{~T}-\mathrm{PC}^{\mathrm{a}}$ & IPM & IPM & IPM-PC & IPM-RFL \\
\hline Bandpass $[\mathrm{keV}]^{\mathrm{b}}$ & $3-50$ & $3-10$ & $3-50$ & $3-50$ & $3-50$ & $3-50$ & $3-50$ & $15-50$ & $3-50$ & $3-50$ \\
\hline$N_{\mathrm{H}}^{f}\left[10^{22} \mathrm{~cm}^{-2}\right]$ & $4.6 \pm 0.4$ & $3.1_{-2.7}^{+1.7}$ & $5.8 \pm 0.3$ & $4.1_{-0.5}^{+0.4}$ & $10.4_{-1.6}^{+1.8}$ & $0.03_{-0.02}^{+4.05}$ & $8.2 \pm 0.3$ & $\cdots$ & $2.6_{-2.6}^{+1.7}$ & $7.7 \pm 0.6$ \\
\hline$N_{\mathrm{H}}^{\mathrm{pc}}\left[10^{23} \mathrm{~cm}^{-2}\right]$ & $\cdots$ & $7.0_{-2.3}^{+2.5}$ & $40 \pm 4$ & $\ldots$ & $\ldots$ & $4.2_{-1.1}^{+1.5}$ & $\ldots$ & $\ldots$ & $4.6_{-1.1}^{+1.5}$ & $\ldots$ \\
\hline$f^{\mathrm{pc}}$ & $\cdots$ & $0.48 \pm 0.07$ & $0.31_{-0.03}^{+0.02}$ & $\cdots$ & $\cdots$ & $0.43_{-0.6}^{+0.07}$ & $\cdots$ & $\ldots$ & $0.4 \pm 0.1$ & $\cdots$ \\
\hline$k T_{\text {low }}[\mathrm{keV}]$ & $\cdots$ & $9.8 \pm 0.9$ & $\ldots$ & $\ldots$ & $1.2_{-0.2}^{+0.3}$ & $2.3_{-0.5}^{+0.8}$ & $\cdots$ & $\ldots$ & $\ldots$ & $\ldots$ \\
\hline$Z^{\mathfrak{c}}$ & $1.3 \pm 0.1$ & $0.36_{-0.07}^{+0.08}$ & $0.93_{-0.09}^{+0.11}$ & $0.9 \pm 0.1$ & $1.2_{-1.3}^{+1.3}$ & $0.7 \pm 0.1$ & $\cdots$ & $\ldots$ & $\cdots$ & 0.5 \\
\hline$N_{\text {low }}\left[10^{-2} \mathrm{~cm}^{-3}\right]$ & $\cdots$ & $7.2 \pm 1.0$ & $\cdots$ & $\cdots$ & $5.1_{-2.3}^{+3.9}$ & $4.2_{-1.4}^{+2.0}$ & $\ldots$ & $\ldots$ & $\cdots$ & $\ldots$ \\
\hline$k T_{\text {high }}[\mathrm{keV}]$ & $\begin{array}{c}20.7 \\
\pm 0.4\end{array}$ & $\cdots$ & $15.6 \pm 0.4$ & $16.7_{-1.9}^{+0.7}$ & $21.1 \pm 0.7$ & $19.7_{-0.8}^{+0.9}$ & $\cdots$ & $\cdots$ & $\ldots$ & $\cdots$ \\
\hline$N_{\text {high }}\left[10^{-2} \mathrm{~cm}^{-3}\right]$ & $3.6 \pm 0.1$ & $\cdots$ & $5.8_{-0.4}^{+0.5}$ & $3.5_{-0.4}^{+0.3}$ & $3.7 \pm 0.1$ & $4.2 \pm 0.2$ & $\cdots$ & $\cdots$ & $\cdots$ & $\cdots$ \\
\hline$M_{\mathrm{WD}}\left[M_{\odot}\right]$ & $\cdots$ & $\ldots$ & $\ldots$ & $\ldots$ & $\ldots$ & $\ldots$ & $\begin{array}{c}0.91 \\
\pm 0.01\end{array}$ & $\begin{array}{c}0.77 \\
\pm 0.03\end{array}$ & $0.80_{-0.02}^{+0.01}$ & $\begin{array}{c}0.78 \\
\pm 0.03\end{array}$ \\
\hline$N_{\text {IPM }}\left[10^{-11}\right]$ & $\cdots$ & $\cdots$ & $\cdots$ & $\cdots$ & $\cdots$ & $\cdots$ & $1.8_{-0.1}^{+0.3}$ & $3.0_{-0.3}^{+0.4}$ & $2.7_{-0.3}^{+0.1}$ & $2.2_{-0.2}^{+0.4}$ \\
\hline$\Omega / 2 \pi$ & $\cdots$ & $\cdots$ & $\cdots$ & $0.6_{-0.2}^{+0.4}$ & $\cdots$ & $\cdots$ & $\cdots$ & $\cdots$ & $\cdots$ & $0.8 \pm 0.2$ \\
\hline$Z_{\mathrm{Fe}}$ & $\cdots$ & $\cdots$ & $\cdots$ & 0.9 & $\cdots$ & $\cdots$ & $\ldots$ & $\ldots$ & $\ldots$ & 0.5 \\
\hline $\cos (\theta)$ & $\ldots$ & $\ldots$ & $\ldots$ & 0.3 & $\cdots$ & $\ldots$ & $\cdots$ & $\cdots$ & $\ldots$ & 0.3 \\
\hline $\mathrm{Fe}-\mathrm{K} \alpha$ line flux ${ }^{\mathrm{d}}$ & $1.1 \pm 0.1$ & $0.9 \pm 0.1$ & $1.0 \pm 0.1$ & $1.0 \pm 0.1$ & $0.9 \pm 0.1$ & $0.8 \pm 0.1$ & $\ldots$ & $\ldots$ & $\ldots$ & $\ldots$ \\
\hline $\mathrm{Fe}-\mathrm{K} \alpha \mathrm{EW}[\mathrm{eV}]$ & $182 \pm 14$ & $88 \pm 15$ & $158 \pm 14$ & $164 \pm 15$ & $123 \pm 14$ & $91 \pm 14$ & $\cdots$ & $\cdots$ & $\ldots$ & $\ldots$ \\
\hline Flux $(2-10 \mathrm{keV})^{\mathrm{e}}$ & 4.3 & 4.3 & 4.3 & 4.3 & 4.2 & 4.4 & 4.0 & 7.1 & 4.2 & 4.0 \\
\hline Flux $(3-50 \mathrm{keV})^{\mathrm{e}}$ & 9.6 & 7.6 & 9.5 & 9.4 & 9.7 & 9.6 & 9.6 & 11.5 & 9.5 & 9.5 \\
\hline$\chi_{\nu}^{2}(\mathrm{dof})$ & $\begin{array}{c}1.33 \\
(596)\end{array}$ & 1.07 (169) & $1.22(594)$ & $1.26(594)$ & 1.18 (594) & $1.10(593)$ & $1.33(546)$ & $0.96(299)$ & $1.14(544)$ & $1.22(544)$ \\
\hline
\end{tabular}

Notes. See Table 2 for the model definitions.

${ }^{a}$ Both APECs are partially covered.

${ }^{\mathrm{b}}$ For all IPM models we ignored the iron line complex (5.5-7.5 keV data bins).

${ }^{\mathrm{c}}$ Abundances $Z$ for low and high $k T$ components are linked.

${ }^{\mathrm{d}}$ The flux unit is $10^{-4} \mathrm{ph} \mathrm{cm}^{-1} \mathrm{~s}^{-1}$. Note that we fixed the line energy to $6.4 \mathrm{keV}$.

e The flux unit is $10^{-11} \mathrm{erg} \mathrm{cm}^{-2} \mathrm{~s}^{-1}$.

Table 8

NuSTAR Spectral Fitting Results of J17303

\begin{tabular}{|c|c|c|c|c|c|c|c|c|c|}
\hline Parameters & $1 \mathrm{~T}$ & 1T-PC & 1T-PC & 1T-RFL & $2 \mathrm{~T}-\mathrm{PC}$ & IPM & IPM-PC & IPM-RFL & IPM-PC-RFL \\
\hline Bandpass $[\mathrm{keV}]^{\mathrm{a}}$ & $3-50$ & $3-10$ & $3-50$ & $3-50$ & $3-50$ & $15-50$ & $3-50$ & $3-50$ & $3-50$ \\
\hline$N_{\mathrm{H}}^{f}\left[10^{22} \mathrm{~cm}^{-2}\right]$ & $7.8_{-0.5}^{+0.6}$ & $2.9_{-2.5}^{+2.4}$ & $4.9 \pm 0.5$ & $3.0 \pm 0.6$ & 4.9 & $\cdots$ & $6.3 \pm 0.6$ & $3.0_{-1.1}^{+1.0}$ & $3.0_{-1.1}^{+1.0}$ \\
\hline$N_{\mathrm{H}}^{\mathrm{pc}}\left[10^{23} \mathrm{~cm}^{-2}\right]$ & $\cdots$ & $11 \pm 3$ & $28 \pm 2$ & $\cdots$ & $26 \pm 2$ & $\cdots$ & $22 \pm 1$ & $\cdots$ & $11_{-2}^{+8}$ \\
\hline$f^{\mathrm{pc}}$ & $\ldots$ & $0.66 \pm 0.08$ & $0.56_{-0.02}^{+0.01}$ & $\cdots$ & $0.59 \pm 0.03$ & $\cdots$ & $0.63 \pm 0.01$ & $\cdots$ & $0.43_{-0.11}^{+0.08}$ \\
\hline$k T_{\text {low }}[\mathrm{keV}]$ & $\cdots$ & $\cdots$ & $\cdots$ & $\cdots$ & $14_{-10}^{+6}$ & $\cdots$ & $\cdots$ & $\cdots$ & $\cdots$ \\
\hline$Z^{\mathrm{b}}$ & 0.3 & 0.3 & $0.3 \pm 0.1$ & $0.3_{-0.1}^{+0.2}$ & $0.3 \pm 0.1$ & $\cdots$ & $\cdots$ & $0.4_{-0.1}^{+0.2}$ & $0.3_{-0.2}^{+0.3}$ \\
\hline$N_{\text {low }}\left[10^{-2} \mathrm{~cm}^{-3}\right]$ & $\cdots$ & $\cdots$ & $\cdots$ & $\cdots$ & $\cdots$ & $\cdots$ & $2.3 \pm 1.0$ & $\cdots$ & \\
\hline$k T_{\text {high }}[\mathrm{keV}]$ & $64.0_{-0.5}$ & $10.2_{-1.1}^{+3.5}$ & $26 \pm 1$ & $41_{-2}^{+3}$ & $43 \pm 12$ & $\cdots$ & $\cdots$ & $\cdots$ & $\cdots$ \\
\hline$N_{\text {high }}\left[10^{-2} \mathrm{~cm}^{-3}\right]$ & $1.94_{-0.05}^{+0.02}$ & $3.79 \pm 0.01$ & $3.5 \pm 0.3$ & $1.3_{-0.2}^{+0.4}$ & $1.7_{-0.5}^{+1.0}$ & $\cdots$ & $\cdots$ & $\cdots$ & $\cdots$ \\
\hline$M_{\mathrm{WD}}\left[M_{\odot}\right]$ & $\cdots$ & $\ldots$ & $\ldots$ & $\ldots$ & $\ldots$ & $1.16 \pm 0.05$ & $0.98 \pm 0.03$ & $1.34 \pm 0.02$ & $1.05_{-0.02}^{+0.03}$ \\
\hline$N_{\text {IPM }}\left[10^{-11}\right]$ & $\ldots$ & $\ldots$ & $\ldots$ & $\ldots$ & $\ldots$ & $0.8 \pm 0.1$ & $1.5 \pm 0.3$ & $0.2 \pm 0.5$ & $0.6_{-0.2}^{+0.3}$ \\
\hline$\Omega / 2 \pi$ & $\cdots$ & $\cdots$ & $\cdots$ & 1.0 & $\cdots$ & $\cdots$ & $\cdots$ & 1.0 & $0.8_{-0.3}^{+0.4}$ \\
\hline$Z_{\mathrm{Fe}}$ & $\cdots$ & $\cdots$ & $\ldots$ & 0.3 & $\ldots$ & $\cdots$ & $\ldots$ & 0.4 & 0.3 \\
\hline $\cos (\theta)$ & $\cdots$ & $\cdots$ & $\cdots$ & $0.95_{-0.06}$ & $\cdots$ & $\cdots$ & $\cdots$ & $0.95_{-0.01}$ & $0.95_{-0.5}$ \\
\hline $\mathrm{Fe}-\mathrm{K} \alpha$ line flux ${ }^{\mathrm{c}}$ & $2.5 \pm 0.5$ & $1.5_{-0.7}^{+0.9}$ & $3.5 \pm 0.5$ & $2.2 \pm 0.5$ & $7.6 \pm 1.1$ & $\cdots$ & $\cdots$ & $\cdots$ & $\cdots$ \\
\hline $\mathrm{Fe}-\mathrm{K} \alpha \mathrm{EW}[\mathrm{eV}]$ & $97 \pm 20$ & $58 \pm 19$ & $146 \pm 22$ & $89 \pm 24$ & $134_{-23}^{+12}$ & $\cdots$ & $\cdots$ & $\cdots$ & $\cdots$ \\
\hline Flux $(2-10 \mathrm{keV})^{\mathrm{d}}$ & 1.7 & 1.8 & 1.7 & 1.7 & 1.7 & 3.9 & 1.6 & 1.7 & 1.7 \\
\hline Flux $(3-50 \mathrm{keV})^{\mathrm{d}}$ & 6.6 & 3.8 & 6.7 & 6.8 & 6.7 & 8.5 & 6.7 & 6.8 & 6.7 \\
\hline$\chi_{\nu}^{2}(\mathrm{dof})$ & $1.90(598)$ & $1.10(168)$ & $1.05(595)$ & $1.03(596)$ & $1.04(594)$ & $1.02(300)$ & $1.00(545)$ & $1.04(544)$ & 0.97 (543) \\
\hline
\end{tabular}

Notes. See Table 2 for the model definitions.

${ }^{\text {a }}$ For all IPM models we ignored the iron line complex (5.5-7.5 keV data bins).

${ }^{\mathrm{b}}$ Abundances $Z$ for low and high $k T$ components are linked.

c The flux unit is $10^{-5} \mathrm{ph} \mathrm{cm}^{-1} \mathrm{~s}^{-1}$. Note that we fixed the line energy to $6.4 \mathrm{keV}$.

${ }^{\mathrm{d}}$ The flux unit is $10^{-11} \mathrm{erg} \mathrm{cm}^{-2} \mathrm{~s}^{-1}$. 
have significant contribution to the Galactic ridge diffuse X-ray emission. Indeed, based on detailed $\mathrm{Fe}$ line diagnostics, $\mathrm{Xu}$ et al. (2016) conclude that the Galactic ridge X-ray emission may consist largely of non-magnetic CVs, not polars, while IPs contribute to a population of brighter and harder sources. Detailed synthetic modeling of the various source populations, taking into account the differences between the ridge and GC regions, such as metallicity, could also prove useful.

\section{SUMMARY}

1. NUSTAR observations of TV Col and J17303 have demonstrated that the most robust masses are obtained using a model that incorporates one-dimensional accretion flow approximations and absorption through the accretion curtain. Restricting the energy band of the model fits to $E \gtrsim 15 \mathrm{keV}$ and ignoring absorption produces equally reliable masses.

2. The NuSTAR observations reveal that the emission from the $\mathrm{CHXE}$ and bright Chandra X-ray point sources $\left(L_{X} \gtrsim 4 \times 10^{32} \mathrm{erg} \mathrm{s}^{-1}\right)$ in the GC is consistent with a large population of IPs with mean mass of $M_{\mathrm{WD}} \sim 0.9 M_{\odot}$. This is a very natural number, since it is in good agreement with the mean mass measured for all CVs by SDSS (Zorotovic et al. 2011).

3. The softer diffuse X-ray emission with $k T \approx 7-8 \mathrm{keV}$ detected by Chandra and XMM-Newton and the harder CHXE have a common origin in massive (compared to isolated WD) IPs.

4. The CHXE XLF must extend down to a luminosity of $L_{X} \lesssim 5 \times 10^{31} \mathrm{erg} \mathrm{s}^{-1}$ in order for the IPs to remain unresolved by NuSTAR. The density of IPs derived from this XLF is consistent with previous XMM-Newton observations, and with crude estimates based on the mass density for the GC, the initial-final mass function for WD, and the probability that these WDs end up in IPs.

5. If the XLFs of the CHXE and the Chandra sources are comparable, $L_{X}^{\min } \lesssim 5 \times 10^{31} \mathrm{erg} \mathrm{s}^{-1}$ implies that $\gtrsim 20 \%-40 \%$ of the Chandra sources are IPs. If the NuSTAR sources observed by Hong et al. (2016) are primarily IPs and/or if the XLF of the CHXE IPs extends down by another factor of $\sim 2$, an overwhelming majority of the Chandra point sources in the GC could be IPs.

6. The Galactic ridge X-ray emission is much softer than the CHXE, and thus may not be composed primarily of IPs. It likely contains an admixture of non-magnetic CVs and polars, with the harder tail comprised of IPs. This is similar to the conclusion of a recent analysis of Fe-line emission from the ridge (Xu et al. 2016), which proposed that non-magnetic CVs are the bulk of the soft X-ray emission and IPs the origin of the harder emission.

This work was supported under NASA Contract No. NNG08FD60C, and made use of data from the NuSTAR mission, a project led by the California Institute of Technology, managed by the Jet Propulsion Laboratory, and funded by the National Aeronautics and Space Administration. We thank the NUSTAR Operations, Software and Calibration teams for support with the execution and analysis of these observations. This research has made use of the NUSTAR Data Analysis Software (NuSTARDAS) jointly developed by the ASI Science Data Center (ASDC, Italy) and the California Institute of
Technology (USA). The authors thank K Mukai and QD Wang for valuable discussions.

\section{APPENDIX}

We present Figures 3, 4 and Tables 7, 8 for the spectral fitting results for TV Col and J17303. The relevant text can be found in Sections 4.1 and 4.2.

\section{REFERENCES}

Aizu, K. 1973, PThPh, 49, 1184

Arnaud, K. A. 1996, in ASP Conf. Ser. 101, Astronomical Data Analysis Software and Systems V, ed. G. H. Jacoby \& J. Barnes (San Francisco, CA: ASP), 17

Barlow, E. J., Knigge, C., Bird, A. J., et al. 2006, MNRAS, 372, 224

Bernardini, F., de Martino, D., Falanga, M., et al. 2012, A\&A, 542, A22

Brunschweiger, J., Greiner, J., Ajello, M., \& Osborne, J. 2009, A\&A, 496, 121

Byckling, K., Mukai, K., Thorstensen, J. R., \& Osborne, J. P. 2010, MNRAS, 408, 2298

Catalán, S., Isern, J., García-Berro, E., \& Ribas, I. 2008, MNRAS, 387, 1693 Cooke, B. A., Ricketts, M. J., Maccacaro, T., et al. 1978, MNRAS, 182, 489 Cropper, M., Ramsay, G., \& Wu, K. 1998, MNRAS, 293, 222

Degenaar, N., Wijnands, R., Cackett, E. M., et al. 2012, A\&A, 545, A49

Dogiel, V. A., Chernyshov, D. O., Kiselev, A. M., et al. 2015, ApJ, 809, 48

Eracleous, M., Halpern, J., \& Patterson, J. 1991, ApJ, 382, 290

Ezuka, H., \& Ishida, M. 1999, ApJS, 120, 277

Ferrario, L., de Martino, D., \& Gänsicke, B. T. 2015, SSRv, arXiv:1504.08072 Ferrario, L., Wickramasinghe, D., Liebert, J., \& Williams, K. A. 2005, MNRAS, 361, 1131

Harrison, F. A., Craig, W. W., Christensen, F. E., et al. 2013, ApJ, 770, 103

Heard, V., \& Warwick, R. S. 2013, MNRAS, 428, 3462

Hellier, C., \& Buckley, D. A. H. 1993, MNRAS, 265, 766

Hong, J., Mori, K., Hailey, C. J., et al. 2016, ApJ, 825, 132

Hong, J., van den Berg, M., Grindlay, J. E., Servillat, M., \& Zhao, P. 2012, ApJ, 746, 165

Hopman, C. 2009, ApJ, 700, 1933

Johnson, S. P., Dong, H., \& Wang, Q. D. 2009, MNRAS, 399, 1429

Kawka, A., Vennes, S., Schmidt, G. D., Wickramasinghe, D. T., \& Koch, R. 2007, ApJ, 654, 499

Kepler, S. O., Kleinman, S. J., Nitta, A., et al. 2007, MNRAS, 375, 1315

Krivonos, R., Revnivtsev, M., Churazov, E., et al. 2007, A\&A, 463, 957

Landi, R., Bassani, L., Dean, A. J., et al. 2009, MNRAS, 392, 630

Magdziarz, P., \& Zdziarski, A. A. 1995, MNRAS, 273, 837

McArthur, B. E., Benedict, G. F., Lee, J., et al. 2001, ApJ, 560, 907

Mewe, R., Gronenschild, E. H. B. M., \& van den Oord, G. H. J. 1985, A\&AS, 62, 197

Mewe, R., Lemen, J. R., \& van den Oord, G. H. J. 1986, A\&AS, 65, 511

Mori, K., Hailey, C. J., Krivonos, R., et al. 2015, ApJ, 814, 94

Mukai, K., Rana, V., Bernardini, F., \& de Martino, D. 2015, ApJL, 807, L30

Muno, M. P., Arabadjis, J. S., Baganoff, F. K., et al. 2004a, ApJ, 613, 1179

Muno, M. P., Baganoff, F. K., Bautz, M. W., et al. 2004b, ApJ, 613, 326

Muno, M. P., Bauer, F. E., Baganoff, F. K., et al. 2009, ApJS, 181, 110

Nauenberg, M. 1972, ApJ, 175, 417

Perez, K., Hailey, C. J., Bauer, F. E., et al. 2015, Natur, 520, 646

Pretorius, M. L., Knigge, C., \& Schwope, A. D. 2013, MNRAS, 432, 570

Pretorius, M. L., \& Mukai, K. 2014, MNRAS, 442, 2580

Rana, V. R., Singh, K. P., Schlegel, E. M., \& Barrett, P. 2004, AJ, 127, 489

Reis, R. C., Wheatley, P. J., Gänsicke, B. T., \& Osborne, J. P. 2013, MNRAS, 430, 1994

Retter, A., Hellier, C., Augusteijn, T., et al. 2003, MNRAS, 340, 679

Revnivtsev, M., Sazonov, S., Churazov, E., et al. 2009, Natur, 458, 1142

Revnivtsev, M., Sazonov, S., Gilfanov, M., Churazov, E., \& Sunyaev, R. 2006, A\&A, 452, 169

Rosen, S. R., Mason, K. O., \& Cordova, F. A. 1988, MNRAS, 231, 549 Scaringi, S., Bird, A. J., Norton, A. J., et al. 2010, MNRAS, 401, 2207

Schödel, R., Feldmeier, A., Kunneriath, D., et al. 2014, A\&A, 566, A47

Smith, R. K., Brickhouse, N. S., Liedahl, D. A., \& Raymond, J. C. 2001, ApJL, 556, L91

Suleimanov, V., Revnivtsev, M., \& Ritter, H. 2005, A\&A, 435, 191

Tout, C. A., Wickramasinghe, D. T., Liebert, J., Ferrario, L., \& Pringle, J. E. 2008, MNRAS, 387, 897

Türler, M., Chernyakova, M., Courvoisier, T. J.-L., et al. 2010, A\&A, 512, A49 
Valinia, A., \& Marshall, F. E. 1998, ApJ, 505, 134

Verner, D. A., Ferland, G. J., Korista, K. T., \& Yakovlev, D. G. 1996, ApJ, 465,487

Vrtilek, S. D., Silber, A., Primini, F., \& Raymond, J. C. 1996, ApJ, 465, 951

Wang, Q. D., Gotthelf, E. V., \& Lang, C. C. 2002, Natur, 415, 148

Wijnen, T. P. G., Zorotovic, M., \& Schreiber, M. R. 2015, A\&A, 577, A143
Wilms, J., Allen, A., \& McCray, R. 2000, ApJ, 542, 914

Xu, X.-j., Wang, Q. D., \& Li, X.-D. 2016, ApJ, 818, 136

Yuasa, T. 2013, PhD thesis, Univ. Tokyo

Yuasa, T., Makishima, K., \& Nakazawa, K. 2012, ApJ, 753, 129

Yuasa, T., Nakazawa, K., Makishima, K., et al. 2010, A\&A, 520, A25

Zorotovic, M., Schreiber, M. R., \& Gänsicke, B. T. 2011, A\&A, 536, A42 